\title{
Solutions of Helmholtz and Schrödinger Equations with Side Condition and Nonregular Separation of Variables ${ }^{\star}$
}

Philip BROADBRIDGE ${ }^{\dagger}$, Claudia M. CHANU ${ }^{\ddagger}$ and Willard MILLER Jr. ${ }^{\S}$

$\dagger$ School of Engineering and Mathematical Sciences, La Trobe University, Melbourne, Australia E-mail: P.Broadbridge@latrobe.edu.au

$¥$ Dipartimento di Matematica G. Peano, Università di Torino, Torino, Italy

E-mail: claudiamaria.chanu@unito.it

$\S$ School of Mathematics, University of Minnesota, Minneapolis, Minnesota, 55455, USA

E-mail: miller@ima.umn.edu

URL: http://www.ima.umn.edu/ miller/

Received September 21, 2012, in final form November 19, 2012; Published online November 26, 2012 http://dx.doi.org/10.3842/SIGMA.2012.089

\begin{abstract}
Olver and Rosenau studied group-invariant solutions of (generally nonlinear) partial differential equations through the imposition of a side condition. We apply a similar idea to the special case of finite-dimensional Hamiltonian systems, namely Hamilton-Jacobi, Helmholtz and time-independent Schrödinger equations with potential on $N$-dimensional Riemannian and pseudo-Riemannian manifolds, but with a linear side condition, where more structure is available. We show that the requirement of $N-1$ commuting second-order symmetry operators, modulo a second-order linear side condition corresponds to nonregular separation of variables in an orthogonal coordinate system, characterized by a generalized Stäckel matrix. The coordinates and solutions obtainable through true nonregular separation are distinct from those arising through regular separation of variables. We develop the theory for these systems and provide examples.
\end{abstract}

Key words: nonregular separation of variables; Helmholtz equation; Schrödinger equation

2010 Mathematics Subject Classification: 35Q40; 35J05

\section{Introduction}

The primary motivation for this paper was the construction by Olver and Rosenau of groupinvariant solutions of (generally nonlinear) partial differential equations through the imposition of a side condition [27, 28]. Various forms of conditional symmetry methods, including nonclassical symmetry reduction, have been applied by several authors, e.g. [1, 5, 6, 10, 21, 26, 29]. We are interested in using linear second-order side conditions and separation of variables to find explicit solutions of Hamilton-Jacobi, Helmholtz, Laplace, wave and heat equations. We exploit the special properties of finite order classical Hamiltonian systems and their quantum analogues represented by Schrödinger equations to obtain new results on separation of variables. The present paper is devoted to Hamilton-Jacobi and Helmholtz or time independent Schrödinger equations with potential but the ideas are clearly applicable to more general Hamiltonian systems and to non-Hamiltonian systems such as diffusion equations that share some features with time-dependent Schrödinger equations.

\footnotetext{
${ }^{\star}$ This paper is a contribution to the Special Issue "Symmetries of Differential Equations: Frames, Invariants
} and Applications". The full collection is available at http://www.emis.de/journals/SIGMA/SDE2012.html 
An example is the Schrödinger equation $H \Psi=E \Psi$ where $H=\Delta+V(\mathbf{x})$ and $\Delta$ is the Laplace-Beltrami operator on some Riemannian or pseudo-Riemannian manifold. We look for solutions of this equation that also satisfy a side condition $S \Psi=0$ where $S$ is some given linear partial differential operator in the variables $\mathbf{x}$. A consistency condition for the existence of nontrivial solutions $\Psi$ is $[H, S]=A S$ for some linear partial differential operator $A$. Moreover a linear differential operator $L$ will be a symmetry operator for $H$, (modulo $S \Psi=0)$ if $[H, L]=$ $B S$ for some linear partial differential operator $B$. To make contact with solutions that are separable in some system of coordinates, we restrict to the case where the symmetries and the side condition are second-order partial differential operators.

A Hamilton-Jacobi analog is the equation $\mathcal{H}=E$ where, in orthogonal coordinates,

$$
\mathcal{H}=\sum_{j=1}^{N} g^{j j}(\mathbf{x}) p_{j}^{2}+V(\mathbf{x}),
$$

which is double the classical Hamiltonian. We look for solutions $u(\mathbf{x})$ of the Hamilton-Jacobi equation, where $p_{j}=\partial_{x^{j}} u$, subject to the side condition $\mathcal{S}(\mathbf{x}, \mathbf{p})=0$. The consistency requirement is the Poisson bracket relation $\{\mathcal{H}, \mathcal{S}\}=\mathcal{A S}$ for some function $\mathcal{A}$ on phase space. Moreover a phase space function $\mathcal{L}$ will be a constant of the motion for $\mathcal{H},($ modulo $\mathcal{S}=0$ ) if $\{\mathcal{H}, \mathcal{L}\}=\mathcal{B S}$ for some phase space function $\mathcal{B}$.

Finding such systems directly from their definition leads to great computational complexity. We explore a new method, based on a generalization of Stäckel form, i.e., a generalization of separable systems corresponding tho a Stäckel matrix, that allows us to generate such systems efficiently.

The second motivation for this paper is the general theory of separation of variables for both linear and nonlinear partial differential equations [17, 20, 23, 24, 25]. In these works the authors point out that there are two types of variable separation: regular and nonregular. Regular separation is the most familiar and was exploited by pioneers such as Stäckel [33] and Eisenhart [12, 14], [13, Appendix 13]. For regular orthogonal separation of a Helmholtz or Schrödinger equation on an $N$-dimensional manifold there are always $N$ separation constants and the associated separable solutions form a basis for the solution space. There is a well developed theory for regular orthogonal separation of these equations, including classification of possible separable coordinate systems in various constant curvature spaces and intrinsic characterizations of the separable systems, see for example [3, 15, 16, 18, 19, 22, 31, 32] in addition to earlier cited references.

For nonregular separation, on the other hand, the number of separation constants is strictly less than $N$ and the separable solutions do not form a basis. Symmetry adapted solutions of partial differential equations are prominent examples of this class, but except for these special solutions there is virtually no structure or classification theory. First attempts of geometric interpretation of nonregular separation are given in $[2,8,9,11]$ where nonregular separation is considered as separation in which the separated solution must satisfy additional constraints that can be seen as side conditions for the equation. In this paper, however, we will show that solutions of Helmholtz and Schrödinger equations with second-order side conditions provide a class of nonregular orthogonal separation of variables that can be characterized intrinsically. Further, each of these systems is associated with a generalized Stäckel matrix and this association enables us to generate nonregular separable systems very easily.

In Sections 2 and 3 we review the Stäckel construction for regular additive separation of Hamilton-Jacobi equations and regular multiplicative separation and $R$-separation for Helmholtz equations, and make some comments on their geometric characterizations. We also discuss the effect of adding vector and scalar potentials. In Section 4 we introduce a generalized Stäckel matrix with one arbitrary column and show that its use leads to additive separable solutions 
of the Hamilton-Jacobi equation, with a side condition. We express the results in a Hamiltonian formalism. Then in Section 5 we carry out the analogous construction for Helmholtz and Schrödinger eigenvalue equations. Section 7 is the main theoretical contribution of our paper. We show that the requirement of maximal nonregular separation for Hamilton-Jacobi equations is equivalent to separation with a generalized Stäckel matrix and can be characterized geometrically. With some modifications, the same is true of $R$-separation for Helmholtz and Schrödinger equations (though there is still a "generalized Rodrigues form" gap in the geometrical characterization). Section 8 is devoted to examples of nonregular separation and discussion of their various types and significance. We prove a "no go" theorem to the effect that nonregular $R$-separation does not occur for the Helmholtz (or Schrödinger) equation with no potential or scalar potential on a 2D Riemannian or pseudo-Riemannian manifold. However 2D nonregular separation can occur for equations with vector or magnetic potentials. Section 8.3 develops the theory for two-dimensional systems with vector potential. The self adjoint Schrödinger equation for a charged particle in two spatial dimensions, interacting with a classical electromagnetic field, again has no new separable coordinate systems that are obtainable from a generalised Stäckel matrix. However, nonself-adjoint equations such as the analogous solute transport equation with first-order convective terms replacing magnetic potential terms, do indeed have new nonregular separable coordinate systems.

We provide examples of nonregular $R$-separation for various zero potential, scalar potential and vector potential 3D systems: Euclidean space, Minkowski space and nonzero constant curvature space, including a Euclidean space example due to Sym [34] of nonregular separation with two side conditions. The final Section 9 sums up our conclusions and points the way for future research on nonregular separation. The coordinate systems and solutions for true nonregular separation, i.e., nonregular separation for which regular separation doesn't occur, are distinct from those for regular separation and further study of their scope and significance is in order.

\section{Review of regular orthogonal separation for the Hamilton-Jacobi equation $\mathcal{H}=E$}

Write the Hamilton-Jacobi equation in terms of $u_{i}=\partial_{i} u(\mathbf{x})$, as

$$
\mathcal{H} \equiv \sum_{i=1}^{N} H_{i}^{-2} u_{i}^{2}+V(\mathbf{x})=E .
$$

Here, the metric in the orthogonal coordinates $x^{i}$ is $d s^{2}=\sum_{i=1}^{N} H_{i}^{2}\left(d x^{i}\right)^{2}$. We want to obtain additive separation, so that $\partial_{j} u_{i} \equiv \partial_{j} \partial_{i} u=0$ for $i \neq j$. Requiring that the solution $u$ depends on $n$ parameters $\left(\lambda_{1}, \ldots, \lambda_{N}\right)$ implies the existence of separation equations in the form

$$
u_{i}^{2}+v_{i}\left(x^{i}\right)+\sum_{j=1}^{N} s_{i j}\left(x^{i}\right) \lambda_{j}=0, \quad i=1, \ldots, N, \quad \lambda_{1}=-E .
$$

Here $\partial_{k} s_{i j}\left(x^{i}\right)=0$ for $k \neq i$ and $\operatorname{det}\left(s_{i j}\right) \neq 0$. We say that $S=\left(s_{i j}\right)$ is a Stäckel matrix. Set $T=S^{-1}$.

Then (2.1) can be recovered from (2.2) provided $H_{j}^{-2}=T^{1 j}$ and $V=\sum_{j} v_{j} T^{1 j}$. The quadratic forms $\mathcal{L}^{\ell}=\sum_{j=1}^{N} T^{\ell j}\left(u_{j}^{2}+v_{j}\right)$ satisfy $\mathcal{L}^{\ell}=-\lambda_{\ell}$ for a separable solution. Furthermore, setting $u_{i}=p_{i}$, we claim

$$
\left\{\mathcal{L}^{\ell}, \mathcal{L}^{j}\right\}=0, \quad \ell \neq j
$$


where $\{\mathcal{H}, \mathcal{K}\}=\sum_{i=1}^{N}\left(\partial_{x^{i}} \mathcal{K} \partial_{p_{i}} \mathcal{H}-\partial_{x^{i}} \mathcal{H} \partial_{p_{i}} \mathcal{K}\right)$ is the Poisson Bracket. Thus the $\mathcal{L}^{\ell}, 2 \leq \ell \leq N$, are constants of the motion for the Hamiltonian $\mathcal{H}=\mathcal{L}^{(1)}$. For the proof of (2.3) one notes that

$$
\sum_{j=1}^{N} T^{\ell j} s_{j k}\left(x^{j}\right)=\delta_{\ell k}
$$

Differentiating this identity with respect to $x^{i}$, we find

$$
\sum_{j=1}^{N} \partial_{i} T^{\ell j} s_{j k}\left(x^{j}\right)+T^{\ell i} s_{i k}^{\prime}\left(x^{i}\right)=0
$$

so

$$
\partial_{i} T^{\ell j}=-T^{\ell i} \sum_{k=1}^{N} s_{i k}^{\prime} T^{k j}
$$

We substitute this expression into the left hand side of (2.3) and obtain the desired result after a routine computation.

\section{Review of the Stäckel procedure for the Helmholtz or Schrödinger equation}

We can perform an analogous construction of eigenfunctions for a Helmholtz operator, using the Stäckel matrix $S$. We demand eigenfunctions of $H$ in the separated form $\Psi=\prod_{j=1}^{N} \Psi^{(j)}\left(x^{j}\right)$ and depending on the maximal number of parameters. Then, the separation equations are of the form

$$
\partial_{\ell}^{2} \Psi+f_{\ell}\left(x^{\ell}\right) \partial_{\ell} \Psi+\left(v_{\ell}\left(x^{\ell}\right)-\sum_{j=1}^{N} s_{\ell j}\left(x^{\ell}\right) \lambda_{j}\right) \Psi=0, \quad \ell=1, \ldots, N
$$

for suitable functions $f_{\ell}, v_{\ell}$ to be determined. Thus we have the eigenvalue equations

$$
L_{k} \Psi \equiv \sum_{\ell=1}^{N} T^{k \ell}\left(\partial_{\ell}^{2}+f_{\ell} \partial_{\ell}+v_{\ell}\right) \Psi=\lambda_{k} \Psi, \quad k=1, \ldots, N
$$

where $L_{1}=H$. Based on our calculations of the preceding section, we can establish the commutation relations

$$
\left[L_{s}, L_{t}\right]=0 .
$$

More generally we can consider $R$-separation for a general Helmholtz equation. In local coordinates $z^{j}$ on an $N$-dimensional pseudo-Riemannian manifold this equation takes the invariant form

$$
H \Theta \equiv\left(\Delta_{N}+\sum_{j=1}^{N} F^{j} \partial_{j}+V\right) \Theta=E \Theta
$$


where

$$
\Delta_{N} \equiv \frac{1}{\sqrt{g}} \sum_{j, k=1}^{N} \partial_{j}\left(g^{j k} \sqrt{g} \partial_{k}\right)
$$

is the Laplace-Beltrami operator. We say that this equation is $R$-separable in local orthogonal coordinates $x^{j}$ if there is a fixed nonzero function $R(\mathbf{x})$ such that (3.2) admits solutions

$$
\Theta=\exp (R) \Psi=\exp (R) \prod_{j=1}^{N} \Psi^{(j)}\left(x^{j}\right),
$$

where $\Psi$ is a regular separated solution, i.e., it satisfies the separation equations (3.1). In this case the symmetry operators are $\tilde{L}_{k}=\exp (R) L_{k} \exp (-R)$ and equations (3.1) become

$$
\partial_{\ell}^{2} \Theta+\left(f_{\ell}\left(x^{\ell}\right)-2 \partial_{\ell} R\right) \partial_{\ell} \Theta+\left(v_{\ell}\left(x^{\ell}\right)-\partial_{\ell \ell} R+\left(\partial_{\ell} R\right)^{2}-\sum_{j=1}^{N} s_{\ell j}\left(x^{\ell}\right) \lambda_{j}\right) \Theta=0
$$

$\ell=1, \ldots, N$. Then we have

$$
\left[\tilde{L}_{s}, \tilde{L}_{t}\right]=0
$$

where $\tilde{L}_{1}=H$.

Now consider the case

$$
\tilde{H} \Theta \equiv\left(\Delta_{N}+V\right) \Theta=E \Theta,
$$

i.e., the case where there is no magnetic field, and $V$ is real. We can define an inner product on the space of $C^{\infty}$ real valued functions $f^{(1)}(\mathbf{z}), f^{(2)}(\mathbf{z})$ with compact support in $\mathbb{R}^{N}$, with respect to which $\tilde{H}$ is formally self-adjoint

$$
\left\langle f^{(1)}, f^{(2)}\right\rangle=\int_{\mathbb{R}^{N}} f^{(1)}(\mathbf{z}) f^{(2)}(\mathbf{z}) \sqrt{g(\mathbf{z})} d \mathbf{z} .
$$

If $L=\sum_{j, k=1}^{N} a^{j k}(\mathbf{z}) \partial_{j k}^{2}+\sum_{\ell=1}^{N} h_{\ell}(\mathbf{z}) \partial_{\ell}+W(\mathbf{z})$ is a real symmetry operator then it can be uniquely decomposed as $L=L^{(1)}+L^{(2)}$ where $L^{(1)}$ is formally self-adjoint and $L^{(2)}$ is formally skewadjoint

$$
L^{(1)}=\frac{1}{\sqrt{g}} \sum_{j, k=1}^{N} \partial_{j}\left(a^{j k} \sqrt{g} \partial_{k}\right)+\tilde{W}, \quad L^{(2)}=\frac{1}{\sqrt{g}} \sum_{\ell=1}^{N}\left(\tilde{h}_{\ell} \partial_{\ell}+\frac{1}{2} \partial_{\ell} \tilde{h}\right) .
$$

Moreover, both $L^{(1)}, L^{(2)}$ are symmetry operators. Note that we have $L^{(2)}=0$ unless $\tilde{H}$ admits a first-order symmetry operator.

Suppose the system admits $N$ algebraically independent commuting symmetry operators $\tilde{L}_{s}$ such that the coefficients $\left(a_{s}^{j k}\right)$ of the second-order terms in the symmetries admit a basis of common eigenforms. (Without loss of generality we can restrict to the self-adjoint case $\tilde{L}_{s}=\tilde{L}_{s}^{(1)}$.) Then it is well established [19], from the examination of the third-order terms in the relations $\left[\tilde{L}_{s}, \tilde{L}_{t}\right]=0$ that there is an orthogonal coordinate system $x^{j}$ and corresponding Stäckel matrix $\left(s_{j k}\left(x^{j}\right)\right)$ such that

$$
\tilde{H}=\sum_{j=1}^{N} \frac{1}{h} \partial_{j}\left(h H_{j}^{-2} \partial_{j}\right)+V, \quad h=H_{1} H_{2} \cdots H_{N} .
$$


Since the metric is in Stäckel form, it is straightforward to verify that the symmetries can be rewritten as

$$
\tilde{L}_{s}=\sum_{j=1}^{N} T^{s j}\left(\partial_{j}^{2}+\left(\partial_{j} \frac{h}{S}\right) \partial_{j}+v_{j}\right),
$$

making it evident that the first derivative terms are a gradient. (Here, $S$ is the determinant of the Stäckel matrix.) Thus via an $R$-transform we can express our system in the form

$$
H=\sum_{j=1}^{N} H_{j}^{-2} \partial_{j}^{2}+\hat{V} .
$$

Then the third derivative terms in the commutation relations $\left[L_{s}, L_{t}\right]=0$ are unchanged and the cancellation of second derivative terms is satisfied identically. The first derivative terms just tell us that the transformed potential $\hat{V}$ is a Stäckel multiplier, so that it permits separation in the coordinates $x^{j}$. The zero-th order relation is satisfied identically. Thus the integrable system (3.3) is $R$-separable. Under the same assumptions, but with a magnetic term added, this is no longer necessarily true. It is easy to see that if there is a function $G$ such that $F^{j}=\partial_{j} G$, i.e., if the magnetic potential is a gradient, then the system is again $R$-separable. However, if the magnetic potential is not a gradient then it is no longer necessarily true that integrability implies $R$-separability, even though the second-order terms in the Laplacian admit a common basis of eigenforms. See [4] for some examples.

\section{A generalization of Stäckel form}

We define a $N \times N$ generalized Stäckel matrix by

$$
S=\left(\begin{array}{ccccc}
s_{11}\left(x^{1}\right) & s_{12}\left(x^{1}\right) & \cdots & s_{1, N-1}\left(x^{1}\right) & a_{1}(\mathbf{x}) \\
s_{21}\left(x^{2}\right) & s_{22}\left(x^{2}\right) & \cdots & s_{2, N-1}\left(x^{2}\right) & a_{2}(\mathbf{x}) \\
\cdots & \cdots & \cdots & \cdots & \cdots \\
s_{N 1}\left(x^{N}\right) & s_{N 2}\left(x^{N}\right) & \cdots & s_{N, N-1}\left(x^{N}\right) & a_{N}(\mathbf{x})
\end{array}\right) .
$$

where the $a_{i}$ are arbitrary analytic functions of the variables $x^{1}, \ldots, x^{N}$. We require that $S$ is a nonsingular matrix. Set $T=S^{-1}$. Now we assume existence of separation equations in the form

$$
u_{i}^{2}+v_{i}\left(x^{i}\right)+\sum_{\xi=1}^{N-1} s_{i \xi}\left(x^{i}\right) \lambda_{\xi}=0, \quad i=1, \ldots, N, \quad \lambda_{1}=-E .
$$

(Here Latin indices take values $1, \ldots, N$ and Greek indices take values $1, \ldots, N-1$.) Note that the term with $\lambda_{N}$ is missing. Thus the general separated solution $u$ will depend on $N$ parameters (rather than $N+1$ ), an example of (maximal) nonregular separation. Note that equations (4.2) can be considered as the restriction to the case $\lambda_{N}=0$ of

$$
u_{i}^{2}+v_{i}\left(x^{i}\right)+\sum_{\xi=1}^{N-1} s_{i \xi}\left(x^{i}\right) \lambda_{\xi}+\lambda_{N} a_{i}(\mathbf{x})=0,
$$

which are not separated for $\lambda_{N}=0$. However, any solution of them is a solution of the $N$ equations

$$
\mathcal{L}^{\ell} \equiv \sum_{j=1}^{N} T^{\ell j}\left(u_{j}^{2}+v_{j}\right)=\lambda_{\ell}, \quad 1 \leq \ell \leq N, \quad \mathcal{L}^{N}=0,
$$


where $\mathcal{H}=\mathcal{L}^{1}$ is the Hamiltonian. Hence, by solving (4.2) we get a separated solution of $\mathcal{H}=E$ satisfying also $\mathcal{L}^{N}=0$ as a side condition. This construction shows that separation with a generalized Stäckel matrix means (nonregular) separation with a side condition. In this case, functions (4.3) become our "restricted constants of the motion" $\mathcal{L}^{\alpha}=\lambda_{\alpha}$ (modulo $\mathcal{L}^{N}=0$ ).

Since

$$
\sum_{j=1}^{N} T^{\ell j} s_{j k}=\delta_{\ell k}
$$

differentiating this identity with respect to $x^{i}$, gives

$$
\sum_{j=1}^{N} \partial_{i} T^{\ell j} s_{j \xi}\left(x_{j}\right)+T^{\ell i} s_{i \xi}^{\prime}\left(x^{i}\right)=0
$$

and

$$
\sum_{j=1}^{N}\left(\partial_{i} T^{\ell j} s_{j N}+T^{\ell j} \partial_{i} a_{j}\right)=0
$$

SO

$$
\partial_{i} T^{\ell j}+T^{\ell i} \sum_{\xi=1}^{N-1} s_{i \xi}^{\prime} T^{\xi j}+T^{N j} \sum_{h=1}^{N} T^{\ell h} \partial_{i} a_{h}=0 .
$$

Using this result it is a straightforward computation to verify the Poisson bracket relations:

$$
\left\{\mathcal{L}^{i}, \mathcal{L}^{j}\right\}=\left(\sum_{k, h=1}^{N}\left(T^{i k} T^{j h}-T^{j k} T^{i h}\right) \frac{\partial a_{k}}{\partial x^{h}} p_{h}\right) \mathcal{L}^{N} .
$$

Relations (4.5) can be considered as the consistency conditions that guarantee the $\mathcal{L}^{i}$ are constants of the motion for the Hamiltonian $\mathcal{H}$, modulo the side condition $\mathcal{L}^{N}=0$. We have verified the relations

$$
\left\{\mathcal{L}^{i}, \mathcal{L}^{j}\right\}_{\mathcal{L}^{N}=0}=0, \quad i, j=1, \ldots, N
$$

for $\mathcal{L}^{1}=\mathcal{H}$ and linearly independent quadratic forms $\mathcal{L}^{i}$. Thus our construction has shown that separation with a generalized Stäckel matrix implies the existence of $N$ independent constants of motion in involution (modulo the side condition) diagonalised in the separable coordinates.

We can generalize Eisenhart's treatment of Stäckel form in which he represented the quadratic forms $T^{\ell i}$ in terms of their eigenvalues with respect to the metric $T^{1 j}=H_{j}^{-2}: T^{\ell j}=\rho_{j}^{(\ell)} H_{j}^{-2}$. Here, $\rho_{j}^{(1)}=1$. Then (4.4) can be rewritten as a system of partial differential equations for the $\rho_{j}^{(\ell)}$ :

$$
\partial_{i} \rho_{j}^{(\ell)}+\rho_{j}^{(\ell)} \frac{\partial_{i} H_{j}^{-2}}{H_{j}^{-2}}+\rho_{i}^{(\ell)} H_{i}^{-2} \sum_{\xi=1}^{N-1} s_{i \xi}^{\prime} \rho_{j}^{(\xi)}+\rho_{j}^{(N)} \sum_{h=1}^{N} \rho_{h}^{(\ell)} H_{h}^{-2} \partial_{i} a_{h}=0 .
$$

In the special case $\ell=1$ these equations reduce to

$$
\frac{\partial_{i} H_{j}^{-2}}{H_{j}^{-2}}+H_{i}^{-2} \sum_{\xi=1}^{N-1} s_{i \xi}^{\prime} \rho_{j}^{(\xi)}+\rho_{j}^{(N)} \sum_{h=1}^{N} H_{h}^{-2} \partial_{i} a_{h}=0 .
$$


Substituting this result in (4.6) we obtain

$$
\partial_{i} \rho_{j}^{(\ell)}+\left(\rho_{j}^{(\ell)}-\rho_{i}^{(\ell)}\right) \frac{\partial_{i} H_{j}^{-2}}{H_{j}^{-2}}+\rho_{j}^{(N)} \sum_{h=1}^{N}\left(\rho_{h}^{(\ell)}-\rho_{i}^{(\ell)}\right) H_{h}^{-2} \partial_{i} a_{h}=0 .
$$

Note that if $\partial_{i} a_{h}=0$ for $i \neq h$ then we recover Stäckel form and (4.7) simplifies to Eisenhart's equation $[12,14]$

$$
\partial_{i} \rho_{j}^{(\ell)}+\left(\rho_{j}^{(\ell)}-\rho_{i}^{(\ell)}\right) \frac{\partial_{i} H_{j}^{-2}}{H_{j}^{-2}}=0 .
$$

A way of expressing the identity (4.7) that does not require the introduction of the terms $a_{h}$ is to note that at least one of the $\rho_{j}^{(N)}$, must be nonzero, say for $j=1$. Setting $j=1$ in $(4.7)$ we obtain

$$
\sum_{h=1}^{N}\left(\rho_{h}^{(\ell)}-\rho_{i}^{(\ell)}\right) H_{h}^{-2} \partial_{i} a_{h}=-\frac{1}{\rho_{1}^{(N)}}\left(\partial_{i} \rho_{1}^{(\ell)}+\left(\rho_{1}^{(\ell)}-\rho_{i}^{(\ell)}\right) \frac{\partial_{i} H_{1}^{-2}}{H_{1}^{-2}}\right) .
$$

Substituting this result back into (4.7) we conclude that

$$
\partial_{i} \rho_{j}^{(\ell)}+\left(\rho_{j}^{(\ell)}-\rho_{i}^{(\ell)}\right) \frac{\partial_{i} H_{j}^{-2}}{H_{j}^{-2}}=\frac{\rho_{j}^{(N)}}{\rho_{1}^{(N)}}\left(\partial_{i} \rho_{1}^{(\ell)}+\left(\rho_{1}^{(\ell)}-\rho_{i}^{(\ell)}\right) \frac{\partial_{i} H_{1}^{-2}}{H_{1}^{-2}}\right) .
$$

For future use we remark that if we restrict to the case $\ell=N$ then, for $B_{j}=\rho_{j}^{(N)} / \rho_{1}^{(N)}$, (4.8) becomes

$$
\partial_{i} B_{j}=\left(B_{i}-B_{j}\right) \frac{\partial_{i} H_{j}^{-2}}{H_{j}^{-2}}+B_{j}\left(1-B_{i}\right) \frac{\partial_{i} H_{1}^{-2}}{H_{1}^{-2}}, \quad i, j=1, \ldots, N .
$$

Remark 1. There is an equivalence relation obeyed by generalized Stäckel matrices. If $S$ is the matrix (4.1), then for any nonzero function $f(\mathbf{x})$, the generalized Stäckel matrix

$$
S^{\prime}=\left(\begin{array}{ccccc}
s_{11}\left(x^{1}\right) & s_{12}\left(x^{1}\right) & \cdots & s_{1, N-1}\left(x^{1}\right) & a_{1}(\mathbf{x}) f(\mathbf{x}) \\
s_{21}\left(x^{2}\right) & s_{22}\left(x^{2}\right) & \cdots & s_{2, N-1}\left(x^{2}\right) & a_{2}(\mathbf{x}) f(\mathbf{x}) \\
\cdots & \cdots & \cdots & \cdots & \cdots \\
s_{N 1}\left(x^{N}\right) & s_{N 2}\left(x^{N}\right) & \cdots & s_{N, N-1}\left(x^{N}\right) & a_{N}(\mathbf{x}) f(\mathbf{x})
\end{array}\right)
$$

defines exactly the same Hamilton-Jacobi equation, separation equations and side condition as does $S$.

Remark 2. This construction of Hamilton-Jacobi systems with a side condition can easily be extended to construct systems with two or more side conditions. For example, with two side conditions $\mathcal{L}^{N-1}=0, \mathcal{L}^{N}=0$, the last two columns of the $N \times N$ generalized Stäckel matrix would be arbitrary and the symmetries would be modulo the side conditions:

$$
\left\{\mathcal{L}^{i}, \mathcal{L}^{j}\right\}=A_{i, j} \mathcal{L}^{N}+B_{i, j} \mathcal{L}^{N-1}, \quad i, j=1, \ldots, N
$$

In a similar fashion nonregular separability of Helmholtz equations with multiple linear side conditions can be defined. 


\section{Generalized Stäckel form for the Helmholtz equation}

Now we perform an analogous construction of eigenfunctions for a Helmholtz-like operator, using the same generalized Stäckel matrix $S$. We want eigenfunctions of the separated form $\Psi=\prod_{j=1}^{N} \Psi^{(j)}\left(x^{j}\right)$. We take separation equations in the form

$$
\partial_{\ell}^{2} \Psi+f_{\ell}\left(x^{\ell}\right) \partial_{\ell} \Psi+\left(v_{\ell}\left(x^{\ell}\right)-\sum_{\alpha=1}^{N-1} s_{\ell \alpha}\left(x^{\ell}\right) \lambda_{\alpha}\right) \Psi=0, \quad \ell=1, \ldots, N .
$$

Then we have the eigenvalue equations

$$
L^{\beta} \Psi \equiv \sum_{\ell=1}^{N} T^{\beta \ell}\left(\partial_{\ell}^{2}+f_{\ell} \partial_{\ell}+v_{\ell}\right) \Psi=\lambda_{\beta} \Psi, \quad \beta=1, \ldots, N-1,
$$

and the side condition

$$
L^{N} \Psi \equiv \sum_{\ell=1}^{N} T^{N \ell}\left(\partial_{\ell}^{2}+f_{\ell} \partial_{\ell}+v_{\ell}\right) \Psi=0
$$

We take $L^{1}=H, \lambda_{1}=E$, so $-\frac{1}{2} H$ is the standard Hamiltonian operator.

Let

$$
X_{\ell}=\partial_{\ell}^{2}+f_{\ell} \partial_{\ell}+v_{\ell}, \quad Y_{\ell}=\partial_{\ell}^{2}+f_{\ell} \partial_{\ell}
$$

We need to compute the commutator $\left[L^{\alpha}, L^{j}\right]$ for $\alpha=1, \ldots, N-1, j=1, \ldots, N$. Now

$$
\begin{aligned}
L^{\alpha} L^{j}= & \left(\sum_{i} \rho_{i}^{(\alpha)} H_{i}^{-2} X_{i}\right)\left(\sum_{k} \rho_{k}^{(j)} H_{k}^{-2} X_{k}\right)=\sum_{i, k} \rho_{i}^{(\alpha)} \rho_{k}^{(j)} H_{i}^{-2} H_{k}^{-2} X_{i} X_{k} \\
& +\sum_{i, k} \rho_{i}^{(\alpha)} H_{i}^{-2} Y_{i}\left(\rho_{k}^{(j)} H_{k}^{-2}\right) X_{k}+2 \sum_{i, k} \rho_{i}^{(\alpha)} H_{i}^{-2} \partial_{i}\left(\rho_{k}^{(j)} H_{k}^{-2}\right) \partial_{i} X_{k}, \\
L^{j} L^{\alpha}= & \left(\sum_{k} \rho_{k}^{(j)} H_{k}^{-2} X_{k}\right)\left(\sum_{i} \rho_{i}^{(\alpha)} H_{i}^{-2} X_{i}\right)=\sum_{i, k} \rho_{k}^{(j)} \rho_{i}^{(\alpha)} H_{k}^{-2} H_{i}^{-2} X_{k} X_{i} \\
& +\sum_{i, k} \rho_{k}^{(j)} H_{k}^{-2} Y_{k}\left(\rho_{i}^{(\alpha)} H_{i}^{-2}\right) X_{i}+2 \sum_{i, k} \rho_{k}^{(j)} H_{k}^{-2} \partial_{k}\left(\rho_{i}^{(\alpha)} H_{i}^{-2}\right) \partial_{k} X_{i},
\end{aligned}
$$

SO

$$
\begin{aligned}
{\left[L^{\alpha}, L^{j}\right]=} & \sum_{i, k}\left(\rho_{i}^{(\alpha)} Y_{i}\left(\rho_{k}^{(j)} H_{k}^{-2}\right)-\rho_{i}^{(j)} Y_{i}\left(\rho_{k}^{(\alpha)} H_{k}^{-2}\right)\right) H_{i}^{-2} X_{k} \\
& +2 \sum_{i, k}\left(\rho_{i}^{(\alpha)} \partial_{i}\left(\rho_{k}^{(j)} H_{k}^{-2}\right)-\rho_{i}^{(j)} \partial_{i}\left(\rho_{k}^{(\alpha)} H_{k}^{-2}\right)\right) H_{i}^{-2} \partial_{i} X_{k} .
\end{aligned}
$$

Using (4.7) we can establish the identities

$$
\begin{aligned}
& \left(\rho_{i}^{(\alpha)} \partial_{i}\left(\rho_{k}^{(j)} H_{k}^{-2}\right)-\rho_{i}^{(j)} \partial_{i}\left(\rho_{k}^{(\alpha)} H_{k}^{-2}\right)\right) H_{i}^{-2} \partial_{i} \\
& \quad=\rho_{k}^{(N)} H_{k}^{-2} H_{i}^{-2} \sum_{h=1}^{N}\left(\rho_{h}^{(\alpha)} \rho_{i}^{(j)}-\rho_{i}^{(\alpha)} \rho_{h}^{(j)}\right) H_{h}^{-2}\left(\partial_{i} a_{h}\right) \partial_{i},
\end{aligned}
$$




$$
\rho_{k}^{(N)} H_{k}^{-2} \partial_{i} F=-\left(\rho_{i}^{(N)} \partial_{i} H_{k}^{-2}+\rho_{k}^{(N)} H_{k}^{-2} \sum_{h=1}^{N}\left(\rho_{i}^{(N)}-\rho_{h}^{(N)}\right) H_{h}^{-2} \partial_{i} a_{h}\right) F+\partial_{i}\left(\rho_{k}^{(N)} H_{k}^{-2} F\right),
$$

for any function $F$, and

$$
\begin{aligned}
\left(\rho_{i}^{(\alpha)} Y_{i}\left(\rho_{k}^{(j)} H_{k}^{-2}\right)-\rho_{i}^{(j)} Y_{i}\left(\rho_{k}^{(\alpha)} H_{k}^{-2}\right)\right) H_{i}^{-2} \\
=2 \rho_{i}^{(N)} H_{i}^{-2} \partial_{i} H_{k}^{-2} \sum_{h=1}^{N}\left(\rho_{h}^{(\alpha)} \rho_{i}^{(j)}-\rho_{h}^{(j)} \rho_{i}^{(\alpha)}\right) H_{h}^{-2} \partial_{i} a_{h} \\
\quad+\rho_{k}^{(N)} H_{k}^{-2} H_{i}^{-2}\left(\rho_{i}^{(j)} \partial_{i}\left(\sum_{h=1}^{N} \rho_{h}^{(\alpha)} H_{h}^{-2} \partial_{i} a_{h}\right)-\rho_{i}^{(\alpha)} \partial_{i}\left(\sum_{h=1}^{N} \rho_{h}^{(j)} H_{h}^{-2} \partial_{i} a_{h}\right)\right) \\
\quad+\rho_{k}^{(N)} H_{k}^{-2} H_{i}^{-2}\left(\sum_{h=1}^{N}\left(\rho_{i}^{(N)}-\rho_{h}^{(N)}\right) H_{h}^{-2} \partial_{i} a_{h}\right)\left(\sum_{h=1}^{N}\left(\rho_{i}^{(j)} \rho_{h}^{(\alpha)}-\rho_{i}^{(\alpha)} \rho_{h}^{(j)}\right) H_{h}^{-2} \partial_{i} a_{h}\right) .
\end{aligned}
$$

Thus,

$$
\begin{aligned}
{\left[L^{\alpha}, L^{j}\right]=} & \sum_{i=1}^{N} H_{i}^{-2}\left(\left(2 \sum_{h=1}^{N}\left(\rho_{i}^{(j)} \rho_{h}^{(\alpha)}-\rho_{i}^{(\alpha)} \rho_{h}^{(j)}\right) H_{h}^{-2} \partial_{i} a_{h}\right)\right. \\
& \times\left(-\frac{1}{2} \sum_{h=1}^{N}\left(\rho_{i}^{(N)}-\rho_{h}^{(N)}\right) H_{h}^{-2} \partial_{i} a_{h}+\partial_{i}\right)+\left(\sum_{h=1}^{N}\left(\rho_{i}^{(N)}-\rho_{h}^{(N)}\right) H_{h}^{-2} \partial_{i} a_{h}\right) \\
& \left.\times\left(\sum_{h=1}^{N}\left(\rho_{i}^{(j)} \rho_{h}^{(\alpha)}-\rho_{i}^{(\alpha)} \rho_{h}^{(j)}\right) H_{h}^{-2} \partial_{i} a_{h}\right)\right) L^{N}=F_{\alpha j} L^{N},
\end{aligned}
$$

where $F_{\alpha j}$ is a first-order partial differential operator.

We see that there is no obstruction to lifting our classical nonregular separation to the operator case. A difficulty occurs, however, when we try to write the pure operator part of $H$ as a Laplace-Beltrami operator on a Riemannian manifold. Then there is an obstruction, a generalized Robertson condition, to be worked out. Also we need to examine the effect of permitting $R$-separation.

\section{Maximal nonregular separation as regular separation with a side condition}

Another way to approach the classical Hamilton-Jacobi problem is to use the Kalnins-Miller method for variable separation $[17,25]$ and consider maximal nonregular separation as regular separation with a side condition. (Here the nonregular separation is maximal in the sense that with a single side condition the number of separation constants is the maximum possible for nonregular separation, i.e., just 1 less than that for regular separation.) We look for additively separable solutions of the equation

$$
\sum_{i=1}^{N} H_{i}^{-2} u_{i}^{2}+V=E
$$

with the side condition

$$
\sum_{i=1}^{N} L_{i}^{-2} u_{i}^{2}+W=0
$$


i.e., solutions $u$ such that $u_{i j}=0$ for $i \neq j$. From (6.1) we find

$$
u_{j j}=-\frac{V_{j}+\sum_{i} \partial_{j} H_{i}^{-2} u_{i}^{2}}{2 H_{j}^{-2} u_{j}},
$$

and from (6.2)

$$
u_{j j}=-\frac{W_{j}+\sum_{i} \partial_{j} L_{i}^{-2} u_{i}^{2}}{2 L_{j}^{-2} u_{j}} .
$$

These expressions must be equal modulo the side condition (6.2), so

$$
\frac{V_{j}+\sum_{i} \partial_{j} H_{i}^{-2} u_{i}^{2}}{2 H_{j}^{-2} u_{j}}=\frac{W_{j}+\sum_{i} \partial_{j} L_{i}^{-2} u_{i}^{2}}{2 L_{j}^{-2} u_{j}}+\nu_{j}\left(\sum_{i} L_{i}^{-2} u_{i}^{2}+W\right),
$$

for some functions $\nu_{j}$. Similarly, equations derived from $u_{j j k}=0$ for $j \neq k$ must hold modulo the side condition. Requiring that all of the above equations hold identically, i.e., requiring that we have regular separation modulo the side condition we eventually obtain the conditions that

1. There are functions $\omega_{j \ell}=\omega_{\ell j}$ for all $j \neq \ell$ such that

$$
-\partial_{j \ell} L_{i}^{-2}+\frac{\partial_{j} L_{\ell}^{-2}}{L_{\ell}^{-2}} \partial_{\ell} L_{i}^{-2}+\frac{\partial_{\ell} L_{j}^{-2}}{L_{j}^{-2}} \partial_{j} L_{i}^{-2}+\omega_{j \ell} L_{i}^{-2}=0
$$

and

$$
-W_{j \ell}+W_{\ell} \frac{\partial_{j} L_{\ell}^{-2}}{L_{\ell}^{-2}}+W_{j} \frac{\partial_{\ell} L_{j}^{-2}}{L_{j}^{-2}}+\omega_{j \ell} W=0
$$

for all $i=1, \ldots, N$. (If all the $L_{\ell}$ are nonzero, this means that the $L_{i}^{-2}$ are in conformal Stäckel form, i.e., an arbitrary function times a Stäckel form matrix, and that $W$ is a conformal Stäckel form potential $[23,24]$.)

2. There are functions $\tau_{j}, j=1, \ldots, N$ such that

$$
\frac{\partial_{j} H_{i}^{-2}}{H_{j}^{-2}}=\frac{\partial_{j} L_{i}^{-2}}{L_{j}^{-2}}+\tau_{j} L_{i}^{-2}
$$

for all $i=1, \ldots, N$, and

$$
\frac{V_{j}}{H_{j}^{-2}}=\frac{W_{j}}{L_{j}^{-2}}+\tau_{j} W
$$

3. Let $C_{i j}$ be the second-order differential operator acting on functions $f$ by

$$
C_{i j}(f)=\partial_{i j} f-\frac{\partial_{j} H_{i}^{-2}}{H_{i}^{-2}} \partial_{i} f-\frac{\partial_{i} \ln H_{j}^{-2}}{H_{j}^{-2}} \partial_{j} f .
$$

There are functions $\mu_{j \ell}=\mu_{\ell j}$ for all $j \neq \ell$ such that

$$
C_{j \ell}\left(H_{i}^{-2}\right)=\mu_{j \ell} L_{i}^{-2}
$$

and

$$
C_{j \ell}(V)=\mu_{j \ell} W
$$

for all $j, \ell=1, \ldots, N$, with $j \neq \ell$. 


\section{Maximal nonregular separation $\Rightarrow$ generalized Stäckel form}

We have shown that the first row of the inverse of a generalized Stäckel matrix with arbitrary $N$-th column is an orthogonal metric $H_{i}^{-2}$ whose associated Hamilton-Jacobi equation admits nonregular separation on the hypersurfaces given by the level set $\mathcal{L}^{N}=0$ of a function $\mathcal{L}^{N}$, quadratic in momentum variables, which is a constrained first integral on the same level set and whose components are the $N$-th row of the inverse of the generalized Stäckel matrix. Now we prove the converse, i.e., that if an orthogonal geodesic Hamiltonian is separable in orthogonal coordinates on the level set of a quadratic first integral, then it is a row of the inverse of a generalized metric and the quadratic coefficients of $\mathcal{L}^{N}$ are the $N$-th row of the generalized Stäckel matrix. Our starting point here is the geometrical framework of regular separation of variables: a complete separated solution of the Hamilton-Jacobi equation is a foliation parametrized by $N$ parameters for the integral manifold of the distribution generated by the $N$ vector fields $D_{i}=$ $\partial_{x^{i}}+R_{i} \partial_{y_{i}}$ where the $R_{i}$ are determined by the condition that the $D_{i}$ are tangent to $\mathcal{H}=$ const. The classical Levi-Civita conditions [22] represent the integrability conditions of the distribution.

In our case we need this distribution to be integrable only on the submanifold $S$ defined by $\mathcal{L}^{N}=0$. We also need the vector fields to be tangent to the submanifold $S$ (closely related to the compatibility of the side condition). Our first step will be to write the differential conditions that in this case play the role of the Levi-Civita condition for regular separation. In this case they mix $\mathcal{H}$ and $\mathcal{L}^{\mathcal{N}}$. Moreover, they include also the condition that $D_{i}$ are tangent to $\mathcal{L}^{N}=0$. We will show that these equations are exactly the equations for nonregular separation derived in Section 6 . Then we will relate these conditions to the existence of some more intrinsic geometrical object for the eigenvalues of Killing tensors (associated with quadratic in the momenta constants of motion), whose integrability conditions are equivalent to the Levi-Civita conditions.

Finally, we will construct the family of quadratic "first integrals" $\mathcal{L}^{h}=\sum_{j=1}^{N} T^{h j} u_{j}^{2}$ commuting and constant for the motion on $\mathcal{L}^{N}=0$ diagonalized in the coordinates we are considering, and show that the inverse of the matrix of the components $T^{h j}$ is a generalized Stäckel matrix. Then we will extend our analysis to prove the corresponding results for multiplicative separation or $R$-separation of the Helmholtz or time independent Schrödinger equations.

\subsection{Differential conditions for nonregular separation on a quadratic first integral leaf}

As in Section 6 we consider a natural Hamiltonian in orthogonal coordinates $\mathbf{x}=\left(x^{i}\right)$ on the cotangent bundle of a $N$-dimensional Riemannian manifold $Q$ with Hamiltonian

$$
\mathcal{H}=\mathcal{L}^{1}=\sum_{i=1}^{N} H_{i}^{-2} p_{i}^{2}+V(\mathbf{x}),
$$

and a function $\mathcal{L}^{N}$ which is also quadratic in the momenta $\left(p_{i}\right)$ and diagonalized in the same coordinates

$$
\mathcal{L}^{N}=\sum_{i=1}^{N} \rho_{i}^{(N)} H_{i}^{-2} p_{i}^{2}+W(\mathbf{x}),
$$

where $\rho_{i}^{(N)}$ are the eigenvalues with respect to the metric $H_{i}^{-2}$, i.e., $L_{i}=\rho_{i}^{(N)} H_{i}^{-2}$. We want to study the existence of separated solutions $u$ of the Hamilton-Jacobi equation

$$
\sum_{i=1}^{N} H_{i}^{-2} u_{i}^{2}+V(\mathbf{x})=E, \quad u_{i}=\partial_{i} u,
$$


with the side condition, or constraint,

$$
\sum_{i=1}^{N} \rho_{i}^{(N)} H_{i}^{-2} u_{i}^{2}+W(\mathbf{x})=0
$$

Recall that the conditions for nonregular separation are (6.4), (6.5), (6.6), (6.7), (6.9), (6.10) for $L_{i}=\rho_{i}^{(N)} H_{i}^{-2}$ and some functions $\omega_{\ell j}, \tau_{j}, \mu_{j \ell}$. However, in this case, we can avoid the introduction of additional unknown functions, since we can solve the equation $\mathcal{L}^{N}=0$ with respect to a momentum variable $u_{i}$ (as for instance $u_{1}^{2}=u_{1}^{2}\left(x^{j}, u_{\alpha}\right)$ with $\alpha=2, \ldots, N$ ) so an expression vanishes on $\mathcal{L}^{N}=0$ if and only if it vanishes for all $u_{\alpha}$ for $\alpha=2, \ldots, N$ after the substitution of $u_{1}$ by $u_{1}\left(x^{j}, u_{\alpha}\right)$. This simplifies very much the task of finding equivalent conditions such as the link with generalized Stäckel matrices and it is possible only because we are assuming orthogonal coordinates.

The conditions (6.4), (6.5), (6.6), (6.7), (6.9), (6.10) are equivalent (supposing without loss of generality that $\left.\rho_{1}^{(N)} \neq 0\right)$ to imposing that for

$$
u_{1}^{2}=-\frac{W}{\rho_{1}^{(N)} H_{1}^{-2}}-\sum_{\alpha=2}^{N} \frac{\rho_{\alpha}^{(N)} H_{\alpha}^{-2}}{\rho_{1}^{(N)} H_{1}^{-2}} u_{\alpha}^{2}
$$

the expressions (6.4)-(6.10) vanish for all values of $u_{\alpha}^{2}$. Even easier, inserting (7.3) in (6.3) we find that the coefficient of $\nu_{j}$ vanishes and equating coefficients of $u_{\alpha}^{2}$ we get

$$
\frac{\partial_{j} \rho_{\alpha}^{(N)} H_{\alpha}^{-2}}{\rho_{\alpha}^{(N)} H_{\alpha}^{-2}}-\frac{\rho_{j}^{(N)}}{\rho_{\alpha}^{(N)}} \frac{\partial_{j} H_{\alpha}^{-2}}{H_{\alpha}^{-2}}=\frac{\partial_{j} \rho_{1}^{(N)} H_{1}^{-2}}{\rho_{1}^{(N)} H_{1}^{-2}}-\frac{\rho_{j}^{(N)}}{\rho_{1}^{(N)}} \frac{\partial_{j} H_{1}^{-2}}{H_{1}^{-2}}=\frac{\partial_{j} W}{W}-\frac{\rho_{j}^{(N)}}{W} \partial_{j} V .
$$

Similarly we find

$$
\frac{C_{i j}\left(H_{\alpha}^{-2}\right)}{\rho_{\alpha}^{(N)} H_{\alpha}^{-2}}=\frac{C_{i j}\left(H_{1}^{-2}\right)}{\rho_{1}^{(N)} H_{1}^{-2}}=\frac{C_{i j}(V)}{W} .
$$

In all these equations we follow the convention that the vanishing of a factor $\rho_{\alpha}^{(N)}$ or $W$ in a denominator in one of expressions (7.4), (7.5), implies that the numerator vanishes.

Note that (7.5) and (7.4) give conditions for separation of the geodesic Hamiltonian with $V=W=0$, with additional conditions that $V$ and $W$ must satisfy. Supposing for simplicity for the moment $V=W=0$, we can rewrite (7.5) and (7.4) as

$$
\begin{aligned}
& \frac{C_{i j}\left(H_{\alpha}^{-2}\right)}{H_{\alpha}^{-2}}=\frac{\rho_{\alpha}^{(N)}}{\rho_{1}^{(N)}} \frac{C_{i j}\left(H_{1}^{-2}\right)}{H_{1}^{-2}}, \\
& \partial_{i} \frac{\rho_{\alpha}^{(N)}}{\rho_{1}^{(N)}}=\left(\frac{\rho_{i}^{(N)}}{\rho_{1}^{(N)}}-\frac{\rho_{\alpha}^{(N)}}{\rho_{1}^{(N)}}\right) \frac{\partial_{i} H_{\alpha}^{-2}}{H_{\alpha}^{-2}}+\frac{\rho_{\alpha}^{(N)}}{\rho_{1}^{(N)}}\left(1-\frac{\rho_{i}^{(N)}}{\rho_{1}^{(N)}}\right) \frac{\partial_{i} H_{1}^{-2}}{H_{1}^{-2}},
\end{aligned}
$$

which are the necessary and sufficient conditions. Equations (7.7) can be interpreted as a first-order system in the $N-1$ unknowns $B_{\alpha}=\frac{\rho_{\alpha}^{(N)}}{\rho_{1}^{(N)}}$.

Proposition 1. A geodesic Hamiltonian $\mathcal{H}$ admits nonregular separation on the submanifold $\mathcal{L}^{N}=0$ in a given orthogonal coordinate system if and only if the functions $B_{k}=\frac{\rho_{k}^{(N)}}{\rho_{1}^{(N)}}(k=$ $1, \ldots, N)$ satisfy

$$
\partial_{i} B_{k}=\left(B_{i}-B_{k}\right) \frac{\partial_{i} H_{k}^{-2}}{H_{k}^{-2}}+B_{k}\left(1-B_{i}\right) \frac{\partial_{i} H_{1}^{-2}}{H_{1}^{-2}} .
$$


Proof. By rewriting the necessary and sufficient conditions (7.6) and (7.7) in terms of the $N$ functions $B_{k}=B_{1}, B_{\alpha}$ with $B_{1}=1$ we get

$$
\frac{C_{i j}\left(H_{k}^{-2}\right)}{H_{k}^{-2}}=B_{k} \frac{C_{i j}\left(H_{1}^{-2}\right)}{H_{1}^{-2}}
$$

and (7.8). However, it is a straightforward calculation that (7.9) is a differential consequence of $(7.8)$, indeed we have

$$
\partial_{i} \partial_{j} B_{k}-\partial_{j} \partial_{i} B_{k}=\left(B_{i}-B_{j}\right)\left(\frac{C_{i j}\left(H_{k}^{-2}\right)}{H_{k}^{-2}}-B_{k} \frac{C_{i j}\left(H_{1}^{-2}\right)}{H_{1}^{-2}}\right) .
$$

Note that the system of equations (7.8) coincides with (4.9), verifying again that separation with a generalized Stäckel matrix is nonregular.

Proposition 2. A natural Hamiltonian $\mathcal{H}$ admits nonregular separation on the submanifold $\mathcal{L}^{N}=0$ in a given orthogonal coordinate system only if the ratios

$$
\frac{C_{i j}\left(H_{\alpha}^{-2}\right) H_{1}^{-2}}{C_{i j}\left(H_{1}^{-2}\right) H_{\alpha}^{-2}}
$$

are independent of $i$ and $j$ and the eigenvalues of the quadratic function $\mathcal{L}^{N}$ are proportional to them

$$
B_{\alpha}=\frac{\rho_{\alpha}^{(N)}}{\rho_{1}^{(N)}}=\frac{C_{i j}\left(H_{\alpha}^{-2}\right) H_{1}^{-2}}{C_{i j}\left(H_{1}^{-2}\right) H_{\alpha}^{-2}} .
$$

Remark 3. The function $\mathcal{L}^{N}$ is naturally defined up to a multiplicative factor $f$ on $Q$ : if an expression is zero on $\mathcal{L}^{N}=0$ then it is also zero on $f \mathcal{L}^{N}=0$ for all functions on $T^{*} Q$, but, since we are interested on quadratic in the momenta functions, we can normalize $f$ on $Q$. (From the Stäckel matrix point of view this corresponds to the multiplication of the $N$-th column by $f$. Hence, equations (7.9) determine the unique (up to a factor) quadratic hypersurface where separation could occur. Indeed, equations (7.10) are the complete integrability conditions for the first-order PDE system (7.8). These conditions are identically satisfied for all $\left(B_{k}, x^{i}\right)$ on an open subset of $\mathbb{C}^{2 n}$ only if $C_{i j}\left(H_{k}^{-2}\right)=0$, that is only if regular separation occurs. However, a single solution $B_{k}=B_{k}\left(x^{i}\right)$ could exist, provided it satisfies the original conditions (7.8), that is if it takes the form (7.11), or with all $B_{k}$ equal, that is for $\mathcal{L}^{N}=\mathcal{H}$. In this case we get separation of the null equation $\mathcal{H}=0$ (in which case a column of the Stäckel matrix was arbitrary, so the result is consistent with the known one).

Let us examine the relation of this kind of separation with the generalized Stäckel matrix.

Theorem 1. Suppose the natural Hamiltonian (7.1) admits $N-2$ other functions $\mathcal{L}^{2}, \ldots, \mathcal{L}^{N-1}$, quadratic in the momenta such that

1) $\mathcal{L}^{1}=\mathcal{H}, \mathcal{L}^{2}, \ldots, \mathcal{L}^{N-1}, \mathcal{L}^{N}$ are pointwise independent,

2) $\mathcal{L}^{1}=\mathcal{H}, \mathcal{L}^{2}, \ldots, \mathcal{L}^{N-1}, \mathcal{L}^{N}$ are constants of the motion, modulo $\mathcal{L}^{N}$, that is

$$
\left.\left\{\mathcal{H}, \mathcal{L}^{k}\right\}\right|_{\mathcal{L}^{N}=0}=0
$$

3) the quadratic terms of $\mathcal{L}^{1}=\mathcal{H}, \mathcal{L}^{2}, \ldots, \mathcal{L}^{N-1}, \mathcal{L}^{N}$ are diagonal in the coordinates $\left(x^{i}\right)$, that is

$$
\mathcal{L}^{\ell}=\sum_{i=1}^{N} \rho_{i}^{(\ell)} H_{i}^{-2}\left(u_{i}^{2}+v_{i}(\mathbf{x})\right), \quad \rho_{i}^{(1)}=1 \quad \forall i
$$


Then,

1) $\partial_{j} v_{i}=0$ for $j \neq i$ and the eigenvalues $\rho_{i}^{(\ell)}$ of $\mathcal{L}^{\ell}$ satisfy the following generalization of the Eisenhart conditions

$$
\partial_{i} \rho_{\alpha}^{(\ell)}+\left(\rho_{\alpha}^{(\ell)}-\rho_{i}^{(\ell)}\right) \frac{\partial_{i} H_{\alpha}^{-2}}{H_{\alpha}^{-2}}=\frac{\rho_{\alpha}^{(N)}}{\rho_{1}^{(N)}}\left(\partial_{i} \rho_{1}^{(\ell)}+\left(\rho_{1}^{(\ell)}-\rho_{i}^{(\ell)}\right) \frac{\partial_{i} H_{1}^{-2}}{H_{1}^{-2}}\right) ;
$$

2) the inverse matrix of $T^{\ell i}=\left(\rho_{i}^{(\ell)} H_{i}^{-2}\right)$ is a generalized Stäckel matrix with the last column made of arbitrary functions of $N$ variables $a_{1}(\mathbf{x}), \ldots, a_{n}(\mathbf{x})$;

3) the Hamilton Jacobi equation (7.2) admits a maximal nonregular separated solution on $\mathcal{L}^{N}=0$ depending on $N-1$ parameters;

4) the $N$ functions $\mathcal{L}^{1}=\mathcal{H}, \mathcal{L}^{2}, \ldots, \mathcal{L}^{N-1}, \mathcal{L}^{N}$ are in involution on $S$, that is

$$
\left.\left\{\mathcal{L}^{\ell}, \mathcal{L}^{k}\right\}\right|_{\mathcal{L}^{N}=0}
$$

Proof. 1) By inserting (7.3) in the Poisson brackets

$$
\begin{aligned}
\left\{\mathcal{L}^{\ell}, \mathcal{H}\right\}= & \sum_{i} 2 H_{i}^{-2} u_{i}\left(\sum_{k=1}^{N}\left(\partial_{i}\left(\rho_{k}^{(\ell)} H_{k}^{-2}\right)-\rho_{i}^{(\ell)} \partial_{i} H_{k}^{-2}\right) u_{k}^{2}\right. \\
& \left.+\sum_{k=1}^{N}\left(\partial_{i}\left(\rho_{k}^{(\ell)} H_{k}^{-2} v_{k}\right)-\rho_{i}^{(\ell)} \partial_{i}\left(H_{k}^{-2} v_{k}\right)\right)\right)
\end{aligned}
$$

we get that $\partial_{i} v_{k}=0$ for $i \neq k$ and that $\rho_{k}^{(\ell)}$ satisfies the generalized Eisenhart conditions (7.12).

2) Since the quadratic diagonal functions are pointwise independent, the matrix $T^{\ell i}$ has a nonzero determinant and therefore it admits an inverse $s_{k h}$ such that

$$
T^{\ell k} s_{k h}=\delta_{h}^{\ell}
$$

(we partially follow the proof of equation (4.7) and a proof of the Eisenhart theorem given in [11]). We want to show that $\partial_{i} s_{h \xi}=0$ for all $i \neq h$ and $\xi \neq N$ if and only if $\rho_{j}^{(\ell)}=T^{\ell j} / T^{1 j}$ satisfy the generalized Eisenhart conditions (7.12). Differentiating (7.13) with respect to $x^{i}$ we get

$$
\sum_{k}\left(\partial_{i} T^{\ell k} s_{k h}+T^{\ell k} \partial_{i} s_{k h}\right)=0
$$

that is

$$
\begin{aligned}
& \sum_{k}\left(\left(H_{k}^{-2} \partial_{i} \rho_{k}^{(\ell)}+\rho_{k}^{(\ell)} \partial_{i} H_{k}^{-2}\right) s_{k h}+\rho_{k}^{(\ell)} H_{k}^{-2} \partial_{i} s_{k h}\right)=0, \quad \ell \neq 1, \\
& \sum_{k}\left(\partial_{i} H_{k}^{-2} s_{k h}+H_{k}^{-2} \partial_{i} s_{k h}\right)=0, \quad \ell=1 .
\end{aligned}
$$

Adding (7.15) multiplied by $-\rho_{i}^{(\ell)}$ to equation (7.14) we get

$$
\sum_{k}\left(\left(H_{k}^{-2} \partial_{i} \rho_{k}^{(\ell)}+\left(\rho_{k}^{(\ell)}-\rho_{i}^{\ell}\right) \partial_{i} H_{k}^{-2}\right) s_{k h}+\left(\rho_{k}^{(\ell)}-\rho_{i}^{(\ell)}\right) H_{k}^{-2} \partial_{i} s_{k h}\right)=0, \quad \ell \neq 1 .
$$


Let us suppose that $\partial_{i} s_{k h}=0$ for all $i \neq k$ and $h \neq N$ and $\partial_{i} s_{k N}=\partial_{i} a_{h}$, by multiplying (7.16) by $T^{h j}$, we get

$$
\sum_{k}\left(H_{k}^{-2} \partial_{i} \rho_{k}^{(\ell)}+\left(\rho_{k}^{(\ell)}-\rho_{i}^{(\ell)}\right) \partial_{i} H_{k}^{-2}\right) \delta_{j}^{k}+\sum_{h} \sum_{k}\left(\left(\rho_{k}^{(\ell)}-\rho_{i}^{(\ell)}\right) H_{k}^{-2} \partial_{i} s_{k h}\right) T^{h j}=0,
$$

that is

$$
H_{j}^{-2} \partial_{i} \rho_{j}^{(\ell)}+\left(\rho_{j}^{(\ell)}-\rho_{i}^{(\ell)}\right) \partial_{i} H_{j}^{-2}+T^{N j} \sum_{k}\left(\left(\rho_{k}^{(\ell)}-\rho_{i}^{(\ell)}\right) H_{k}^{-2} \partial_{i} a_{k}\right)=0 .
$$

Writing (7.17) for $j=1$ and $j=\alpha$ and by combining them in order to eliminate the common factor $\sum_{k}\left(\left(\rho_{k}^{(\ell)}-\rho_{i}^{(\ell)}\right) H_{k}^{-2} \partial_{i} a_{k}\right)$, we get (7.12). Conversely, let us suppose that (7.12) hold; this means that the quantities

$$
\frac{H_{k}^{-2} \partial_{i} \rho_{k}^{(\ell)}+\left(\rho_{k}^{(\ell)}-\rho_{k}^{(\ell)}\right) \partial_{i} H_{k}^{-2}}{T^{N k}}
$$

are independent of $k$, that is $H_{k}^{-2} \partial_{i} \rho_{k}^{(\ell)}+\left(\rho_{k}^{(\ell)}-\rho_{k}^{(\ell)}\right) \partial_{i} H_{k}^{-2}=T^{N k} Q_{i}^{\ell}$. Therefore (7.16) becomes

$$
T^{N k} Q_{i}^{\ell} s_{k h}+\sum_{k}\left(\left(\rho_{k}^{(\ell)}-\rho_{i}^{(\ell)}\right) H_{k}^{-2} \partial_{i} s_{k h}\right)=\delta_{h}^{N} Q_{i}^{\ell}+\sum_{k}\left(\left(\rho_{k}^{(\ell)}-\rho_{i}^{(\ell)}\right) H_{k}^{-2} \partial_{i} s_{k h}\right)=0 .
$$

If we fix the values of $h$ and $i$, then the above equations are a linear system of $N-1$ equations $(\ell=2, \ldots, N)$ in $N-1$ unknown $\left.\partial_{i} s_{k h} k=1, \ldots, N, k \neq h\right)$. For $h \neq N$ the system is homogeneous and the matrix of coefficient $M^{\ell k}=\left(\rho_{k}^{(\ell)}-\rho_{i}^{(\ell)}\right) H_{k}^{-2}$ has nonzero determinant (see [11]). Hence, the only solution is $\partial_{i} s_{k h}=0$ for $h \neq N$.

3) If equations (7.12) hold, they hold in particular for $\ell=N$ and we get (7.7) which are proven to be equivalent to conditions (7.8) that are necessary and sufficient for the nonregular separation with the side condition $\mathcal{L}^{N}=0$. Further, the conditions (7.4) on $V, W$ are exactly the consistency conditions that must be satisfied by $V, W$ in order that $\left\{\mathcal{H}, \mathcal{L}^{N}\right\}=0$ hold on $\mathcal{L}^{N}=0$, and conditions (7.5) are exactly the integrability conditions for $V, W$ that must be satisfied if $\left\{\mathcal{H}, \mathcal{L}^{N}\right\}=0$ is to hold.

4) This is essentially the computation in Section 4.

Theorem 2. If the $N$ pointwise independent functions

$$
\mathcal{L}^{1}=\mathcal{H}, \mathcal{L}^{2}, \ldots, \mathcal{L}^{N-1}, \mathcal{L}^{N}
$$

are in involution on $\mathcal{L}^{N}=0$ and admit common eigenvectors, i.e., if the quadratic forms in the momenta associated with $\mathcal{L}^{\ell}-\rho^{(\ell)} \mathcal{H}$ have $N$ common eigenvectors, then the eigenvectors are normal: there exist orthogonal coordinates $\left(x^{i}\right)$ such that the $N$ functions are simultaneously diagonalized.

Remark 4. This is essentially the converse of the results of Section 4. It shows that separation with a side condition implies the existence of a generalized Stäckel matrix that defines the separation.

Proof. Apply Theorem 7.5 of [2] with $M_{a b}=K^{N}$, where $K^{N}$ is the symmetric 2-tensor associated with $\mathcal{L}^{N}$.

Remark 5. The functions $\mathcal{L}^{\ell}$ are defined up to multiples of $\mathcal{L}^{N}$ and the conformal Killing tensors are defined up to multiple of the metric tensor. Indeed, $\mathcal{L}^{\ell}+f^{\ell} \mathcal{L}^{N}$ have the same eigenvalues of $\mathcal{L}^{\ell}$ (provided that the $\mathcal{L}^{\ell}$ have common eigenvectors) and they are in involution when restricted to $\mathcal{L}^{N}=0$ :

$$
\left\{\mathcal{L}^{\ell}+f^{\ell} \mathcal{L}^{N}, \mathcal{L}^{k}+f^{k} \mathcal{L}^{N}\right\}=\mathcal{Q} \mathcal{L}^{N}
$$


Now we close the logical loop. Suppose the conditions (7.8)-(7.10) of maximal nonregular separation are satisfied and consider the linear system of equations

$$
\partial_{i} \rho_{j}+\left(\rho_{j}-\rho_{i}\right) \frac{\partial_{i} H_{j}^{-2}}{H_{j}^{-2}}=B_{j}\left(\partial_{i} \rho_{1}+\left(\rho_{1}-\rho_{i}\right) \frac{\partial_{i} H_{1}^{-2}}{H_{1}^{-2}}\right), \quad i, j=1, \ldots, N,
$$

for the unknowns $\left(\rho_{1}, \ldots, \rho_{N}\right)$. Remark 5 suggests that this system has solutions for any choice of $\rho_{1}$. Therefore, we choose the function $\rho_{1}$ arbitrarily and consider (7.18) as a system of $N(N-1)$ independent differential equations for the unknowns $\left(\rho_{2}, \ldots, \rho_{N}\right)$. It is then a straightforward exercise to verify that the integrability conditions for (7.18) are satisfied identically, due to the nonregular separation conditions. We already know the two solutions $(1,1, \ldots, 1)$ and $\left(B_{2}, \ldots, B_{N}\right)$. Indeed about any regular point $\mathbf{x}_{0}$ we can find a unique solution such that $\rho_{j}\left(\mathbf{x}_{0}\right)=\beta_{j}, 2 \leq j \leq N$ for any choice of constants $\beta_{j}$. For example we could take $\rho_{1} \equiv 1$ and define $N-1$ linearly independent solutions by choosing the $\beta_{j}$ accordingly. Then we could take $\rho_{1} \equiv 0$ and find another solution such that the full $N$ solutions form a basis. This basis determines a generalized Stäckel matrix.

It remains to consider the potential terms, the given functions $V$ and $W$. Using the functions $\rho_{i}^{(\ell)}$ of the generalized Stäckel matrix computed above we define functions

$$
\mathcal{L}^{\ell}=\sum_{i=1}^{N} \rho_{i}^{(\ell)} H_{i}^{-2} u_{i}^{2}+W^{(\ell)}, \quad \ell=1, \ldots, N-1,
$$

where $V=W^{(1)}$ but the remaining $W^{(\ell)}$ will be determined by imposing the requirement that there exist functions $F_{j}$ linear in the momenta and such that

$$
\left\{\mathcal{H}, \mathcal{L}^{N}\right\}=F_{N} \mathcal{L}^{N}, \quad\left\{\mathcal{H}, \mathcal{L}^{\ell}\right\}=F_{\ell} \mathcal{L}^{N} .
$$

By working out the Poisson brackets, it is straightforward to verify that equations (7.4) are exactly the consistency conditions for the existence of $F_{N}$ and (7.5) are exactly the consistency conditions for the $F_{\ell}$ and the integrability conditions for the existence of the potentials $W^{(\ell)}$.

Now note that since the generalized Stäckel matrix is invertible we can uniquely determine functions $v_{i}(\mathbf{x})$ such that

$$
W^{(j)}=\sum_{i=1}^{N} v_{i} \rho_{i}^{(j)} H_{i}^{-2}, \quad j=1, \ldots, N .
$$

With this observation we have verified all of the assumptions of Theorem 1 . This proves the following result.

Theorem 3. If a natural Hamiltonian $\mathcal{H}$ admits maximal nonregular separation on the submanifold $\mathcal{L}^{N}=0$ in a given orthogonal coordinate system, then the system is separable with a side condition and there exists a generalized Stäckel matrix for the separation.

All of these results for additive nonregular separation of Hamilton-Jacobi equations extend to multiplicative nonregular separation for the Helmholtz (or Schrödinger) equations on the same manifold, but with an obstruction. If a system is nonregular $R$-separable for the Helmholtz equation then it is nonregular separable for the Hamilton-Jacobi equation. However, if the Hamilton-Jacobi equation admits nonregular separation in some coordinate system then there is a "generalized Robertson condition" to be solved to determine for which potentials the Helmholtz equation admits $R$-separation in these coordinates. On the other hand, we can always find some family of vector potentials for which the Helmholtz equation does admit nonregular $R$-separation in the coordinates. That is, it is easy to show that given a nonregular separable system for the Hamilton-Jacobi equation, we can construct a family of vector potentials for which the corresponding Helmholtz equation is nonregular $R$-separable. We give examples in Section 8 . 


\section{Examples of nonregular separability for Hamilton-Jacobi and Helmholtz equations}

\subsection{Examples of restricted regular separation}

The simplest examples of Theorems 2 and 3, and their extensions to the Helmholtz equation, are those for which regular orthogonal separation already occurs. Thus the "generalized" Stäckel matrix (4.1) is a true Stäckel matrix. Consider first the separable Hamilton-Jacobi equation in the form

$$
\mathcal{H} \equiv \sum_{i=1}^{N} H_{i}^{-2} u_{i}^{2}+V(\mathbf{x})=E .
$$

Here, the metric in the orthogonal separable coordinates $x^{i}$ is

$$
d s^{2}=\sum_{i=1}^{N} H_{i}^{2}\left(d x^{i}\right)^{2}
$$

There is an associated Stäckel matrix and constants of the motion $\mathcal{L}^{\ell}, 2 \leq \ell \leq N$, where $\mathcal{H}=\mathcal{L}^{1}$. We apply the side condition $\mathcal{L}^{N^{\prime}} \equiv \mathcal{L}^{N}-\lambda_{N}=0$ where $\lambda_{N}$ is a constant scalar. Note that $\mathcal{L}^{N^{\prime}}$ is also a constant of the motion. Now the separation equations become

$$
u_{i}^{2}+\left(v_{i}\left(x^{i}\right)+\lambda_{N} s_{i N}\left(x^{i}\right)\right)+\sum_{j=1}^{N-1} s_{i j}\left(x^{i}\right) \lambda_{j}=0, \quad i=1, \ldots, N, \quad \lambda_{1}=-E .
$$

Our "restricted constants of the motion" become

$$
\mathcal{L}^{\ell^{\prime}} \equiv \sum_{j=1}^{N} T^{\ell j}\left(u_{j}^{2}+v_{j}+\lambda_{N} s_{j N}\right)
$$

so we have modified the potential. The side conditions are satisfied automatically. Effectively, we have restricted our Hamiltonian system to the $N-1$ dimensional hypersurface $\mathcal{L}^{N}=\lambda_{N}$ and seen that the result is again a Hamiltonian system. Under some circumstances this has a simple interpretation as a separable system on a manifold of one less dimension.

Example 1. Consider the Kepler Hamiltonian

$$
\mathcal{H}=\mathcal{L}^{1}=p_{x}^{2}+p_{y}^{2}+p_{z}^{2}+\frac{\alpha}{r}, \quad r=\sqrt{x^{2}+y^{2}+z^{2}}
$$

in real Euclidean space. The system is separable in spherical coordinates $r, \theta, \phi$ where

$$
\begin{aligned}
& x=r \sin \theta \cos \phi, \quad y=r \sin \theta \sin \phi, \quad z=r \cos \theta, \\
& r \geq 0, \quad 0 \leq \theta \leq \pi, \quad 0 \leq \phi<2 \pi .
\end{aligned}
$$

Here

$$
\mathcal{H}=p_{r}^{2}+\frac{\mathcal{L}^{2}}{r^{2}}+\frac{\alpha}{r}, \quad \mathcal{L}^{2}=p_{\theta}^{2}+\frac{\mathcal{L}^{3}}{\sin ^{2} \theta}, \quad \mathcal{L}^{3}=p_{\phi}^{2} .
$$

We choose the side condition $\mathcal{L}^{3}=\lambda_{3}$. Then the reduced Hamiltonian becomes

$$
\mathcal{H}^{\prime}=p_{r}^{2}+\frac{p_{\theta}^{2}}{r^{2}}+\frac{\lambda_{3}}{r^{2} \sin ^{2} \theta}+\frac{\alpha}{r} .
$$


In terms of the new variables $X=r \cos \theta, Y=r \sin \theta$ this becomes

$$
\mathcal{H}^{\prime}=p_{X}^{2}+p_{Y}^{2}+\frac{\alpha}{\sqrt{X^{2}+Y^{2}}}+\frac{\lambda_{3}}{Y^{2}}
$$

a regular separable system in the first quadrant of the Euclidean plane. In the special case $\lambda_{3}=0$ the singularity on the $X$-axis disappears and we can extend the system to the full punctured plane. Note that this construction is conceptually distinct from simply restricting a trajectory confined to a plane.

A similar construction works for the Helmholtz equation. We want eigenfunctions of the separated form $\Psi=\prod_{j=1}^{N} \Psi^{(j)}\left(x^{j}\right)$. We take separation equations in the form

$$
\partial_{\ell}^{2} \Psi+f_{\ell}\left(x^{\ell}\right) \partial_{\ell} \Psi+\left(v_{\ell}\left(x^{\ell}\right)-s_{\ell N}\left(x^{\ell}\right) \lambda_{N}-\sum_{\alpha=1}^{N-1} s_{\ell \alpha}\left(x^{\ell}\right) \lambda_{\alpha}\right) \Psi=0, \quad \ell=1, \ldots, N .
$$

Then we have the restricted eigenvalue equations

$$
L^{\beta^{\prime}} \Psi \equiv \sum_{\ell=1}^{N} T^{\beta \ell}\left(\partial_{\ell}^{2}+f_{\ell} \partial_{\ell}+v_{\ell}-s_{\ell N} \lambda_{N}\right) \Psi=\lambda_{\beta} \Psi, \quad \beta=1, \ldots, N-1,
$$

and the side condition

$$
L^{N^{\prime}} \Psi \equiv \sum_{\ell=1}^{N} T^{N \ell}\left(\partial_{\ell}^{2}+f_{\ell} \partial_{\ell}+v_{\ell}-s_{\ell N} \lambda_{N}\right) \Psi=0 .
$$

We take $L^{1^{\prime}}=H^{\prime}$. Effectively, we have restricted our separable quantum system to an eigenspace $\left\{\Psi: L^{N} \Psi=\lambda_{N} \Psi\right\}$ of a symmetry operator. The restriction is again a Hamiltonian system. Again, under some circumstances this has a simple interpretation as a separable quantum system on a manifold of one less dimension.

Example 2. Consider the hydrogen atom Hamiltonian, a constant multiple of

$$
H=L^{1}=\partial_{x}^{2}+\partial_{y}^{2}+\partial_{z}^{2}+\frac{\alpha}{r}, \quad r=\sqrt{x^{2}+y^{2}+z^{2}}
$$

in real Euclidean space. The system is separable in spherical coordinates $r, \theta, \phi$ :

$$
H=\partial_{r}^{2}+\frac{2}{r} \partial_{r}+\frac{1}{r^{2}} L^{2}+\frac{\alpha}{r}, \quad L^{2}=\partial_{\theta}^{2}+\cot \theta \partial_{\theta}+\frac{1}{\sin ^{2} \theta} L^{3}, \quad L^{3}=\partial_{\phi}^{2} .
$$

We choose the side condition $L^{3} \Psi=\lambda_{3} \Psi$. Then the reduced Hamiltonian becomes

$$
H^{\prime}=\partial_{r}^{2}+\frac{2}{r} \partial_{r}+\frac{1}{r^{2}} \partial_{\theta}^{2}+\frac{\cot \theta}{r^{2}} \partial_{\theta}+\frac{\lambda_{3}}{r^{2} \sin ^{2} \theta}+\frac{\alpha}{r} .
$$

Now we make an $R$-transformation $\Psi=R \Theta$ where $R=\frac{1}{r \sqrt{\sin \theta}}$. Then the eigenvalue equation $H^{\prime} \Psi=E \Psi$ becomes

$$
\left(\partial_{r}^{2}+\frac{1}{r^{2}} \partial_{\theta}^{2}+\frac{\lambda_{3}+\frac{1}{4}}{r^{2} \sin ^{2} \theta}+\frac{\frac{1}{4}}{r^{2}}+\frac{\alpha}{r}\right) \Theta=E \Theta, \quad \partial_{\phi}^{2} \Theta=\lambda_{3} \Theta .
$$


Now write $\Theta=\Phi(\phi) \Xi(r, \theta)$. In terms of the new variables $X=r \cos \theta, Y=r \sin \theta$ the energy equation is

$$
\left(\partial_{X}^{2}+\partial_{Y}^{2}+\frac{\alpha}{\sqrt{X^{2}+Y^{2}}}+\frac{\frac{1}{4}}{X^{2}+Y^{2}}+\frac{\lambda_{3}+\frac{1}{4}}{Y^{2}}\right) \Xi=E \Xi,
$$

a regular separable system in the upper half Euclidean plane. If $\lambda_{3}=-1 / 4$ this equation can be extended to the punctured plane and regarded as a modification of the hydrogen atom in the plane. Note, however, that due to the $R$ factor, the usual $L_{2}$ normalization for eigenstates in Euclidean space doesn't restrict to the usual $L_{2}$ normalization for eigenstates in the plane. Thus, in analogy with the virial theorem for the mapping between the Coulomb and pseudoCoulomb problems [36], one needs to check that the bound state spectra are preserved under the restriction.

\subsection{Nonregular separation in $2 \mathrm{D}$ and a "no go" theorem}

Now we take up the issue of true nonregular separation in a coordinate system in which regular separation is impossible. Let us first look at $2 \mathrm{D}$ examples, a very special case. By using the facts that we can always replace a separable coordinate by an invertible function of itself, and we can perform linear transformations on the separation constants without changing the system, we can always assume that the generalized Stäckel matrix looks like

$$
\tilde{S}=\left(\begin{array}{ll}
1 & A(u, v) \\
1 & B(u, v)
\end{array}\right) .
$$

We must require that $A-B \neq 0$ so that the matrix is invertible and that $A B \neq 0$ so that the metric is nondegenerate. Further, by making use of the equivalence relation (4.10) we can put the matrix in canonical form

$$
S=\left(\begin{array}{cc}
1 & 1 \\
1 & f(u, v)
\end{array}\right), \quad \text { with inverse } T=\frac{1}{f-1}\left(\begin{array}{cc}
f & -1 \\
-1 & 1
\end{array}\right),
$$

where $f=B / A$. This form will lead to true nonregular separation unless $f$ can be factored as $f(u, v)=U(u) V(v)$, because in that case we can take $B=V(v), A=1 / U(u)$ and find an equivalent true Stäckel matrix. It is easy to see that this form leads to true nonregular separation for the Hamilton-Jacobi equation. However, in the 2D case the construction fails for Helmholtz equations.

Theorem 4. For a $2 D$ manifold the Helmholtz equation $\Delta \Psi=\lambda \Psi$ never admits true nonregular separation.

Proof. In the 2D case, the Laplacian takes the form

$$
\Delta=\frac{f}{f-1}\left(\partial_{u u}+\frac{1}{2} \frac{f_{u}}{f} \partial_{u}\right)-\frac{1}{f-1}\left(\partial_{v v}-\frac{1}{2} \frac{f_{v}}{f} \partial_{v}\right) .
$$

For nonregular separation it is necessary that $f_{u} / f$ is a function of $u$ alone and $f_{v} / f$ is a function of $v$ alone. Thus $f=U(u) V(v)$ and the system admits regular separation. Similarly if $\Psi=e^{R} \Theta$ for some fixed $R$, then $R$-separation implies that there exist functions $U(u), V(v)$ such that

$$
R_{u}=-\frac{1}{4} \frac{f_{u}}{f}+U(u), \quad R_{v}=\frac{1}{4} \frac{f_{v}}{f}+V(v) .
$$

Since $\partial_{v} R_{u}=\partial_{u} R_{v}$ it follows easily that $\partial_{u v} \ln f=0$. Thus $f=\hat{U}(u) \hat{V}(v)$ and the system must admit regular $R$-separation. 
However, true nonregular separation may occur when vector potentials are included, even in the $2 \mathrm{D}$ case. The self-adjoint Schrödinger equation for a non-relativistic charged particle influenced by a classical electromagnetic 4-potential, still does not admit true nonregular separation. Nonetheless true nonregular separation does occur when the vector potential gives rise to skew-adjoint first-order terms, as in a diffusion-convection equation for transport of solute.

\subsection{Quantum particle in $\mathbb{R}^{2}$ with a magnetic field}

In the Schrödinger picture with Cartesian coordinates, the eigenvalue equation for the quantized Hamiltonian of a non-relativistic charged particle interacting with a classical electromagnetic field, is (e.g. [4])

$$
H \Psi=-\frac{(\hbar)^{2}}{2} \nabla^{2} \Psi-\frac{i \hbar}{2}[\mathbf{A} \cdot \nabla+\nabla \cdot \mathbf{A}(\mathbf{x})] \Psi+\Phi(\mathbf{x}) \Psi=\lambda \Psi .
$$

$\left(A_{1}, A_{2}, 0\right)$ is the vector potential for the magnetic field and $\Phi$ is the scalar potential for the electric field. We consider the equivalent eigenvalue problem

$$
H \Psi=\partial_{1}^{2} \Psi+\partial_{2}^{2} \Psi+\frac{i}{2}\left[a^{1}(\mathbf{x}) \partial_{1}+a^{2}(\mathbf{x}) \partial_{2}+\nabla \cdot \mathbf{a}(\mathbf{x})\right] \Psi+\Phi(\mathbf{x}) \Psi=E \Psi .
$$

We extend this equation to a general curvilinear orthogonal coordinate system. As before,

$$
\Delta=\left[H_{1}^{-2} \partial_{1}^{2}+H_{2}^{-2} \partial_{2}^{2}+H_{1}^{-2} \partial_{1} \log \frac{H_{2}}{H_{1}} \partial_{1}-H_{2}^{-2} \partial_{2} \log \frac{H_{2}}{H_{1}} \partial_{2}\right]
$$

In a general orthogonal coordinate system, the scalar divergence, involving Christoffel symbol $\Gamma$, of vector $\mathbf{a}$, is

$$
\begin{aligned}
\operatorname{div} \mathbf{a} & =\frac{\partial a^{m}}{\partial x^{m}}+a^{m} \Gamma_{i m}^{i}=\frac{1}{\sqrt{g}} \frac{\partial}{\partial x^{i}}\left(a^{i} \sqrt{g}\right) \\
& =\partial_{i} a^{i}+a^{1}\left[\partial_{1} \log H_{1}+\partial_{1} \log H_{2}\right]+a^{2}\left[\partial_{2} \log H_{1}+\partial_{2} \log H_{2}\right] .
\end{aligned}
$$

The eigenvalue equation for the Hamiltonian is

$$
\begin{aligned}
H \Psi= & H_{1}^{-2} \partial_{1}^{2} \Psi+H_{2}^{-2} \partial_{2}^{2} \Psi+\left[H_{1}^{-2} \partial_{1} \log \frac{H_{2}}{H_{1}}\right] \partial_{1} \Psi-\left[H_{2}^{-2} \partial_{2} \log \frac{H_{2}}{H_{1}}\right] \partial_{2} \Psi \\
& +\frac{i}{2}\left[a^{1}(\mathbf{x}) \partial_{1}+\partial_{1} a^{1}(\mathbf{x})+a^{2}(\mathbf{x}) \partial_{2}+\partial_{2} a^{2}(\mathbf{x})\right] \Psi \\
& +\frac{i}{2}\left[a^{1} \partial_{1} \log \left(H_{1}\right)+a^{1} \partial_{1} \log \left(H_{2}\right)+a^{2} \partial_{2} \log \left(H_{1}\right)+a^{2} \partial_{2} \log \left(H_{2}\right)\right] \Psi+\Phi(\mathbf{x}) \Psi=E \Psi .
\end{aligned}
$$

Consider $R$-separation

$$
\Theta=\Psi e^{-R(\mathbf{x})}=\Theta^{(1)}\left(x^{1}\right) \Theta^{(2)}\left(x^{2}\right) .
$$

Then

$$
\begin{aligned}
& {\left[H_{1}^{-2} \partial_{1}^{2}+H_{2}^{-2} \partial_{2}^{2}+H_{1}^{-2}\left\{\partial_{1} \log \frac{H_{2}}{H_{1}}+2 \partial_{1} R\right\} \partial_{1}+H_{2}^{-2}\left\{-\partial_{2} \log \frac{H_{2}}{H_{1}}+2 \partial_{2} R\right\} \partial_{2}\right] \Theta} \\
& +H_{1}^{-2}\left[\partial_{1}^{2} R+\left(\partial_{1} R\right)^{2}+\left(\partial_{1} R\right) \partial_{1} \log \frac{H_{2}}{H_{1}}\right] \Theta \\
& +H_{2}^{-2}\left[\partial_{2}^{2} R+\left(\partial_{2} R\right)^{2}-\left(\partial_{2} R\right) \partial_{1} \log \frac{H_{2}}{H_{1}}\right] \Theta+\Phi(\mathbf{x}) \Theta
\end{aligned}
$$




$$
\begin{aligned}
& +\frac{i}{2}\left[a^{1} \partial_{1} \Theta+a^{2} \partial_{2} \Theta\right]+\frac{i}{2}\left[a^{1} \partial_{1} R+a^{2} \partial_{2} R+\partial_{1} a^{1}+\partial_{2} a^{2}\right] \Theta \\
& +\frac{i}{2} a^{1}\left[\partial_{1} \log H_{1}+\partial_{1} \log H_{2}\right] \Theta+\frac{i}{2} a^{2}\left[\partial_{2} \log H_{1}+\partial_{2} \log H_{2}\right] \Theta=E \Theta .
\end{aligned}
$$

Assume the separation equations

$$
\begin{aligned}
& \partial_{1}^{2} \Theta+\left[f_{1}\left(x^{1}\right)+i g_{1}\left(x^{1}\right)\right] \partial_{1} \Theta+\left[\left[v_{1}\left(x^{1}\right)+i w_{1}\left(x^{1}\right)\right]-S_{11}\left(x^{1}\right) E\right] \Theta=0, \\
& \partial_{2}^{2} \Theta+\left[f_{2}\left(x^{2}\right)+i g_{2}\left(x^{2}\right)\right] \partial_{2} \Theta+\left[\left[v_{2}\left(x^{2}\right)+i w_{2}\left(x^{2}\right)\right]-S_{21}\left(x^{2}\right) E\right] \Theta=0, \quad \Rightarrow \\
& T_{11} \partial_{1}^{2} \Theta+T_{12} \partial_{2}^{2} \Theta+T_{11}\left[f_{1}+i g_{1}\right] \partial_{1} \Theta+T_{12}\left[f_{2}+i g_{2}\right] \partial_{2} \Theta \\
& \quad+\left[T_{11}\left[v_{1}+i w_{1}\right]+T_{12}\left[v_{2}+i w_{2}\right]\right] \Theta=E \Theta, \\
& T_{21} \partial_{1}^{2} \Theta+T_{22} \partial_{2}^{2} \Theta+T_{21}\left[f_{1}+i g_{1}\right] \partial_{1} \Theta+T_{22}\left[f_{2}+i g_{2}\right] \partial_{2} \Theta \\
& \quad+\left[T_{21}\left[v_{1}+i w_{1}\right]+T_{22}\left[v_{2}+i w_{2}\right]\right] \Theta=0,
\end{aligned}
$$

where matrix $T$ is the inverse of generalized Stäckel matrix $S$. In order for the above eigenvalue equation to be identified with the Schrödinger equation, we again require $T_{11}=H_{1}^{-2}$ and $T_{12}=H_{2}^{-2}$, and in addition,

$$
\begin{aligned}
& f_{1}\left(x^{1}\right)=\partial_{1} \log \frac{H_{2}}{H_{1}}+2 \partial_{1} R, \quad f_{2}\left(x^{2}\right)=-\partial_{2} \log \frac{H_{2}}{H_{1}}+2 \partial_{2} R, \\
& T_{11} v_{1}+T_{12} v_{2}= \Phi+H_{1}^{-2} \partial_{1}^{2} R+H_{2}^{-2} \partial_{2}^{2} R+H_{1}^{-2}\left(\partial_{1} R\right)^{2}+H_{2}^{-2}\left(\partial_{2} R\right)^{2} \\
&+H_{1}^{-2}\left(\partial_{1} R\right) \partial_{1} \log \frac{H_{2}}{H_{1}}-H_{2}^{-2}\left(\partial_{2} R\right) \partial_{2} \log \frac{H_{2}}{H_{1}}, \\
& g_{1}\left(x^{1}\right)=\frac{1}{2} H_{1}^{2} a^{1}, \quad g_{2}\left(x^{2}\right)=\frac{1}{2} H_{2}^{2} a^{2}, \\
& T_{11} w_{1}+T_{12} w_{2}= \frac{1}{2}\left[a^{1} \partial_{1} R+a^{2} \partial_{2} R+\partial_{1} a^{1}+\partial_{2} a^{2}\right] \\
&+\frac{1}{2} a^{1}\left[\partial_{1} \log H_{1}+\partial_{1} \log H_{2}\right]+\frac{1}{2} a^{2}\left[\partial_{2} \log H_{1}+\partial_{2} \log H_{2}\right] .
\end{aligned}
$$

From (8.2) we can easily deduce that $\partial_{1} \partial_{2} R=0$ and $\partial_{1} \partial_{2} \log \left(H_{2} / H_{1}\right)=0$. Hence

$$
H_{2} / H_{1}=\Pi_{1}\left(x^{1}\right) \Pi_{2}\left(x^{2}\right) \quad \text { and } \quad R=R_{1}\left(x^{1}\right)+R_{2}\left(x^{2}\right)
$$

for some functions $\Pi_{1}, \Pi_{2}, R_{1}, R_{2}$. Now assuming the canonical form with $H_{1}=1$, we have

$$
a^{1}=2 g_{1}\left(x^{1}\right) \quad \text { and } \quad a^{2}=2 g_{2}\left(x^{2}\right) \Pi_{2}\left(x^{2}\right)^{-2} \Pi_{1}\left(x^{1}\right)^{-2},
$$

whence it follows by separation of variables in (8.3), that

$$
\begin{aligned}
& \Pi_{1}^{2}\left[w_{1}-g_{1} \partial_{1} R_{1}-\partial_{1} g_{1}-g_{1} \partial_{1} \log \Pi_{1}\right]=\gamma_{4}, \\
& \Pi_{2}^{-2}\left[w_{2}-g_{2} \partial_{2} R_{2}-\partial_{2} g_{2}+g_{2} \partial_{2} \log \Pi_{2}\right]=-\gamma_{4},
\end{aligned}
$$

where $\gamma_{4}$ is constant. By integration,

$$
\begin{aligned}
& a^{1}=2 \Pi_{1}^{-1} e^{-R_{1}}\left[\gamma_{5}+\int e^{R_{1}}\left[w_{1} \Pi_{1}-\gamma_{4} \Pi_{1}^{-1}\right] d x^{1}\right], \\
& a^{2}=2 \Pi_{1}^{-2} \Pi_{2}^{-1} e^{-R_{2}}\left[\gamma_{6}+\int e^{R_{2}}\left[w_{2} \Pi_{2}^{-1}+\gamma_{4} \Pi_{2}\right] d x^{2}\right],
\end{aligned}
$$

with $\gamma_{4}, \gamma_{5}$ and $\gamma_{6}$ constant. As a standard test, we first recover the known possibility of separation in polar coordinates in $E^{2}$, with

$$
x^{1}=r, \quad x^{2}=\theta, \quad \Pi_{1}=r, \quad \Pi_{2}=1 .
$$


In (8.2) we may choose $f_{1}=f_{2}=0$ without any loss of generality to the class of allowable scalar and vector potentials. Consequently, from (8.2), $R_{1}=-\frac{1}{2} \log r$ and $R_{2}=0$. This yields the potential fields that admit separation of the Schrödinger equation

$$
\begin{aligned}
& a^{1}=\alpha_{1}(r)=\gamma_{5} r^{-1 / 2}+2 \gamma_{4} r^{-1}+r^{-1 / 2} \int r^{1 / 2} w_{1}(r) d r \\
& a^{2}=\alpha_{2}(\theta) r^{-2}=2 \gamma_{6} r^{-2}+2 r^{-2} \int \gamma_{4}+w_{2}(\theta) d \theta, \quad \Phi=v_{1}(r)+r^{-2} v_{2}(\theta)-\frac{1}{4} r^{-2} .
\end{aligned}
$$

The above form for the scalar potential agrees with that known to allow separation of polar coordinates in the Helmholtz operator [19]. When the vector potential a in (8.1) takes values in $\mathbb{R}^{2}$, the separation condition (8.4) again leads to the conclusion that in this case, generalized Stäckel matrices do not lead to new separable systems.

\subsection{Solute transport}

The possibilities of new separable equations are broadened if we replace the pure imaginary coefficients of first-order terms in (8.1) by first-order terms with real coefficients. Consider a solute diffusion-convection equation in Cartesian coordinates,

$$
\partial_{t} \Psi=H \Psi=\partial_{1}^{2} \Psi+\partial_{2}^{2} \Psi-\left[q^{1}(\mathbf{x}) \partial_{1}+q^{2}(\mathbf{x}) \partial_{2}\right] \Psi-\mu(\mathbf{x}) \Psi .
$$

In this application, $\Psi$ represents the solute concentration, $\mathbf{q}(\mathbf{x})$ represents a steady velocity field of the solvent, and $\mu(\mathbf{x})$ is an adsorption coefficient for removal of solute by the solid substrate of a porous medium or by another (usually solid) component of the mixture. If $\Psi(\mathbf{x})$ satisfies $H \Psi=E \Psi$, then $e^{E t} \Psi(\mathbf{x})$ is a solution of the time dependent solute equation. In standard applications, $E \leq 0$, unless the solid component of the mixture is releasing solute $(\mu(\mathbf{x})<0)$ rather than adsorbing solute. After making the replacements $\mathbf{q}=(-i / 2) \mathbf{a}$ and $\mu(\mathbf{x})=-\Phi(\mathbf{x})-(i / 2) \nabla \cdot \mathbf{a}$, the solute equation is directly analogous to the Schrödinger equation with magnetic field, except that now $q^{j}$ must be real, corresponding to $a^{j}$ being pure imaginary, $a^{j}=-i A^{j}, A^{j}=-2 q^{j}$,

$$
\partial_{t} \Psi=H \Psi=\partial_{1}^{2} \Psi+\partial_{2}^{2} \Psi+\frac{1}{2}\left[A^{1}(\mathbf{x}) \partial_{1}+A^{2}(\mathbf{x}) \partial_{2}+\nabla \cdot \mathbf{A}(\mathbf{x})\right] \Psi+\Phi(\mathbf{x}) \Psi .
$$

In a general orthogonal coordinate system,

$$
\begin{aligned}
H \Psi= & H_{1}^{-2} \partial_{1}^{2} \Psi+H_{2}^{-2} \partial_{2}^{2} \Psi+\left[H_{1}^{-2} \partial_{1} \log \frac{H_{2}}{H_{1}}\right] \partial_{1} \Psi-\left[H_{2}^{-2} \partial_{2} \log \frac{H_{2}}{H_{1}}\right] \partial_{2} \Psi \\
& +\frac{1}{2}\left[A^{1}(\mathbf{x}) \partial_{1}+\partial_{1} A^{1}(\mathbf{x})+A^{2}(\mathbf{x}) \partial_{2}+\partial_{2} A^{2}(\mathbf{x})\right] \Psi+\frac{1}{2}\left[A^{1} \partial_{1} \log \left(H_{1}\right)\right. \\
& \left.+A^{1} \partial_{1} \log \left(H_{2}\right)+A^{2} \partial_{2} \log \left(H_{1}\right)+A^{2} \partial_{2} \log \left(H_{2}\right)\right] \Psi+\Phi(\mathbf{x}) \Psi=E \Psi,
\end{aligned}
$$

consider $R$-separation

$$
\Theta=\Psi e^{-R(\mathbf{x})}=\Psi^{(1)}\left(x^{1}\right) \Psi^{(2)}\left(x^{2}\right) .
$$

Then

$$
\begin{aligned}
& {\left[H_{1}^{-2} \partial_{1}^{2}+H_{2}^{-2} \partial_{2}^{2}+H_{1}^{-2}\left\{\partial_{1} \log \frac{H_{2}}{H_{1}}+2 \partial_{1} R\right\} \partial_{1}+H_{2}^{-2}\left\{-\partial_{2} \log \frac{H_{2}}{H_{1}}+2 \partial_{2} R\right\} \partial_{2}\right] \Theta} \\
& \quad+H_{1}^{-2}\left[\partial_{1}^{2} R+\left(\partial_{1} R\right)^{2}+\left(\partial_{1} R\right) \partial_{1} \log \frac{H_{2}}{H_{1}}\right] \Theta
\end{aligned}
$$




$$
\begin{aligned}
& +H_{2}^{-2}\left[\partial_{2}^{2} R+\left(\partial_{2} R\right)^{2}-\left(\partial_{2} R\right) \partial_{1} \log \frac{H_{2}}{H_{1}}\right] \Theta+\Phi(\mathbf{x}) \Theta \\
& +\frac{1}{2}\left[A^{1} \partial_{1} \Theta+A^{2} \partial_{2} \Theta\right]+\frac{1}{2}\left[A^{1} \partial_{1} R+A^{2} \partial_{2} R+\partial_{1} A^{1}+\partial_{2} A^{2}\right] \Theta \\
& +\frac{1}{2} A^{1}\left[\partial_{1} \log H_{1}+\partial_{1} \log H_{2}\right] \Theta+\frac{1}{2} A^{2}\left[\partial_{2} \log H_{1}+\partial_{2} \log H_{2}\right] \Theta=E \Theta .
\end{aligned}
$$

Assume the separation equations

$$
\begin{aligned}
& \partial_{1}^{2} \Theta+f_{1}\left(x^{1}\right) \partial_{1} \Theta+\left[v_{1}\left(x^{1}\right)-S_{11}\left(x^{1}\right) E\right] \Theta=0, \\
& \partial_{2}^{2} \Theta+f_{2}\left(x^{2}\right) \partial_{2} \Theta+\left[v_{2}\left(x^{2}\right)-S_{21}\left(x^{2}\right) E\right] \Theta=0, \quad \Rightarrow \\
& T_{11} \partial_{1}^{2} \Theta+T_{12} \partial_{2}^{2} \Theta+T_{11} f_{1} \partial_{1} \Theta+T_{12} f_{2} \partial_{2} \Theta+\left[T_{11} v_{1}+T_{12} v_{2}\right] \Theta=E \Theta, \\
& T_{21} \partial_{1}^{2} \Theta+T_{22} \partial_{2}^{2} \Theta+T_{21} f_{1} \partial_{1} \Theta+T_{22} f_{2} \partial_{2} \Theta+\left[T_{21} v_{1}+T_{22} v_{2}\right] \Theta=0,
\end{aligned}
$$

where matrix $T$ is the inverse of generalized Stäckel matrix $S$. In order for the above eigenvalue equation to be identified with the solute equation, we again require $T_{11}=H_{1}^{-2}, T_{12}=H_{2}^{-2}$. Since the first-order terms of the target solute equation no longer have imaginary coefficients, the identification of first-order terms now leads to more general possibilities

$$
H_{1}^{2} q^{1}=-f_{1}\left(x^{1}\right)+\partial_{1} \log \frac{H_{2}}{H_{1}}+2 \partial_{1} R, \quad H_{2}^{2} q^{2}=-f_{2}\left(x^{2}\right)-\partial_{2} \log \frac{H_{2}}{H_{1}}+2 \partial_{2} R .
$$

By differentiating throughout, this implies

$$
\partial_{1} \partial_{2} \log \frac{H_{2}}{H_{1}}+2 \partial_{1} \partial_{2} R-\partial_{2}\left(H_{1}^{2} q^{1}\right)=0, \quad-\partial_{1} \partial_{2} \log \frac{H_{2}}{H_{1}}+2 \partial_{1} \partial_{2} R-\partial_{1}\left(H_{2}^{2} q^{2}\right)=0 .
$$

This system is equivalent to

$$
4 \partial_{1} \partial_{2} R-\partial_{2}\left(H_{1}^{2} q^{1}\right)-\partial_{1}\left(H_{2}^{2} q^{2}\right)=0, \quad 2 \partial_{1} \partial_{2} \log \frac{H_{2}}{H_{1}}-\partial_{2}\left(H_{1}^{2} q^{1}\right)+\partial_{1}\left(H_{2}^{2} q^{2}\right)=0 .
$$

Similarly, identification of the zero-th order terms also leads to a more general condition

$$
\begin{aligned}
T_{11} v_{1}+T_{12} v_{2}= & -\mu+H_{1}^{-2} \partial_{1}^{2} R+H_{2}^{-2} \partial_{2}^{2} R+H_{1}^{-2}\left(\partial_{1} R\right)^{2}+H_{2}^{-2}\left(\partial_{2} R\right)^{2} \\
& +H_{1}^{-2}\left(\partial_{1} R\right) \partial_{1} \log \frac{H_{2}}{H_{1}}-H_{2}^{-2}\left(\partial_{2} R\right) \partial_{2} \log \frac{H_{2}}{H_{1}}-q^{1} \partial_{1} R-q^{2} \partial_{2} R .
\end{aligned}
$$

In principle, the compatible velocity field $\mathbf{q}$ is recovered from (8.5) after substituting these expressions in (8.6) which determines the function $R$.

The condition $H_{2} / H_{1}=\Pi_{1}\left(x^{1}\right) \Pi_{2}\left(x^{2}\right)$, which is the key condition for the possibility of replacement by a regular Stäckel matrix construction, is no longer true in general but occurs only if

$$
\partial_{2}\left(H_{1}^{2} q^{1}\right)-\partial_{1}\left(H_{2}^{2} q^{2}\right)=0
$$

In a description with Cartesian coordinates for Euclidean space, this would be exactly the restriction that the solvent velocity field is irrotational; $\nabla \times \mathbf{q}=\mathbf{0}$. Consider the canonical form

$$
S^{-1}=T=\frac{1}{f(\mathbf{x})-1}\left[\begin{array}{cc}
f & -1 \\
-1 & 1
\end{array}\right] .
$$

Then (8.6) may be written as,

$$
\begin{aligned}
-f v_{1}+v_{2}= & -f \partial_{1}^{2} R+\partial_{2}^{2} R+f\left(\partial_{1} R\right)^{2}-\left(\partial_{2} R\right)^{2}-f \partial_{1} R \partial_{1} \log (-f)+2 f \partial_{1} R \partial_{1} \log (1-f) \\
& -f_{1} \partial_{1} R+\partial_{2} R \partial_{2} \log (-f)-2 \partial_{2} R \partial_{2} \log (1-f)+f_{2} \partial_{2} R+(f-1) \mu .
\end{aligned}
$$

In looking for examples of genuine nonregular separation, we must consider metrics for which $H_{2} / H_{1}$ does not separate in variables $x^{1}$ and $x^{2}$. 
Example 3. We consider an example in 2D Euclidean space. The metric is

$$
\begin{aligned}
& d s^{2}=d x^{2}+d y^{2}=\frac{f(u, v)-1}{f(u, v)} d u^{2}+(1-f(u, v)) d v^{2}, \\
& f(u, v)=-\frac{1}{4}\left(u+v+\sqrt{(u+v)^{2}-4}\right)^{2} .
\end{aligned}
$$

Here

$$
\begin{aligned}
& x=(u+v) \cos (\phi-u), \quad y=(u+v) \sin (\phi-u), \\
& \phi=\frac{1}{2}\left(u+v+\sqrt{(u+v)^{2}-4}\right)-2 \arctan \left(\frac{u+v+\sqrt{(u+v)^{2}-4}}{2}\right),
\end{aligned}
$$

and $|u+v| \geq 2$. The Helmholtz equation with vector and scalar potential takes the form

$$
\left(\Delta_{2}-\frac{f}{f-1}\left(\frac{1}{2} \frac{f_{u}}{f} \partial_{u}-U(u)\right)+\frac{1}{f-1}\left(\frac{-1}{2} \frac{f_{v}}{f} \partial_{v}-V(v)\right)\right) \Psi=E \Psi .
$$

The separation equations are

$$
\left(\partial_{u u}+U(u)-E\right) \Psi^{(1)}(u)=0, \quad\left(\partial_{v v}+V(v)-E\right) \Psi^{(2)}(v)=0,
$$

with $\Psi=\Psi^{(1)}(u) \Psi^{(2)}(v)$.

Now $r^{2}=x^{2}+y^{2}=(u+v)^{2}$, from which the polar coordinate may be taken to be $r=u+v$. Then

$$
x=r \cos (\phi-u), \quad y=r \sin (\phi-u),
$$

implying that the polar angle coordinate is

$$
\phi-u=\theta \quad(\bmod 2 \pi) .
$$

Also

$$
\phi=\frac{1}{2}\left(r+\sqrt{r^{2}-4}\right)-2 \arctan \left(\frac{r+\sqrt{r^{2}-4}}{2}\right) .
$$

Note that $\pm \theta$ is an additive component of each of the variables $u=\phi(r)-\theta$ and $v=r-\phi(r)+\theta$. Therefore physically relevant solutions must be periodic with period $2 \pi$ in each of the variables $u$ and $v$. Taking the simplest case $v^{1}(=U)=0$ and $f^{1}(=V)=0$, direct separation is possible with $R=0$, and from (8.5)

$$
\left(H_{1}\right)^{2} q^{1}=-\left(H_{2}\right)^{2} q^{2}=1 / \sqrt{(u+v)^{2}-4}=\left[r^{2}-4\right]^{-0.5} .
$$

The velocity field is defined on the exterior to the circle of radius 2 centred at the origin. The squared magnitude of velocity is

$$
\left(q^{1}\right)^{2} \mathbf{e}_{1} \cdot \mathbf{e}_{1}+\left(q^{2}\right)^{2} \mathbf{e}_{2} \cdot \mathbf{e}_{2}=H_{1}^{2}\left(q^{1}\right)^{2}+H_{2}^{2}\left(q^{2}\right)^{2}=\frac{1}{r^{2}-4}
$$

After taking $E=-\omega^{2}$, separated solutions for solute concentration may be combined in a Fourier integral

$$
\Psi=\int_{0}^{\infty} A(\omega) e^{-\omega^{2} t} \cos (\omega[u+\delta(\omega)]) \cos (\omega[v+\varepsilon(\omega)]) d \omega,
$$

with amplitude function $A(\omega)$ and phase functions $\delta(\omega), \varepsilon(\omega)$. 


\subsection{Nonregular separation in more than 2 dimensions}

In dimensions greater than two, both true nonregular separation and $R$-separation occur even without an added vector potential.

Example 4. This is a 3D Minkowski space example. The metric is

$$
d s^{2}=d t^{2}-d x^{2}-d y^{2}=-\frac{4 w^{2}}{\left(u^{2}-v^{2}\right)^{2}}\left(d u^{2}+d v^{2}\right)+d w^{2}=H_{u}^{2} d u^{2}+H_{v}^{2} d v^{2}+H_{w}^{2} d w^{2} .
$$

Here,

$$
t=\frac{w}{u^{2}-v^{2}}\left(\frac{1}{4}+\left(u^{2}+v^{2}\right)^{2}\right), \quad x=\frac{w}{u^{2}-v^{2}}\left(\frac{1}{4}-\left(u^{2}+v^{2}\right)^{2}\right), \quad y=\frac{2 w u v}{u^{2}-v^{2}},
$$

and we require $u, v$ real and $w>0,|u|>|v|$.

The Helmholtz equation in 3D Minkowski space is

$$
\Delta_{3} \Theta=E \Theta,
$$

where

$$
\Delta_{3}=\frac{\left(u^{2}-v^{2}\right)^{2}}{4 w^{2}}\left(-\partial_{u u}-\partial_{v v}+\frac{4 w^{2}}{\left(u^{2}-v^{2}\right)^{2}} \partial_{w w}+\frac{8 w}{\left(u^{2}-v^{2}\right)^{2}} \partial_{w}\right) .
$$

We look for $R$-separable solutions

$$
\Theta=e^{R} \Psi=e^{R} \Psi^{(1)}(u) \Psi^{(2)}(v) \Psi^{(3)}(w), \quad e^{R}=\frac{1}{w} .
$$

By direct calculation we can establish the operator identity

$$
e^{-R} \Delta_{2} e^{R}-E=H_{u}^{-2}\left(\partial_{u u}+\lambda_{2}\right)+H_{v}^{-2}\left(\partial_{v v}-\lambda_{2}\right)+H_{w}^{-2}\left(\partial_{w w}-\frac{1}{w^{2}}-E\right) .
$$

Thus the generalized Stäckel matrix and its inverse are

$$
S=\left(\begin{array}{ccc}
0 & 1 & 1+\frac{\left(u^{2}-v^{2}\right)^{2}}{4 w^{2}} \\
0 & -1 & -\frac{\left(u^{2}-v^{2}\right)^{2}}{4 w^{2}} \\
1 & 0 & \frac{\left(u^{2}-v^{2}\right)^{2}}{4 w^{2}}
\end{array}\right), \quad T=\left(\begin{array}{ccc}
-\frac{\left(u^{2}-v^{2}\right)^{2}}{4 w^{2}} & -\frac{\left(u^{2}-v^{2}\right)^{2}}{4 w^{2}} & 1 \\
-\frac{\left(u^{2}-v^{2}\right)^{2}}{4 w^{2}} & -1-\frac{\left(u^{2}-v^{2}\right)^{2}}{4 w^{2}} & 0 \\
1 & 1 & 0
\end{array}\right) .
$$

In terms of the $\Psi$ functions we have

$$
\begin{aligned}
& L^{1} \Psi=\left(-\frac{\left(u^{2}-v^{2}\right)^{2}}{4 w^{2}}\left(\partial_{u u}+\partial_{v v}\right)+\left(\partial_{w w}-\frac{1}{w^{2}}\right)\right) \Psi=E \Psi \\
& L^{2} \Psi=\left(-\frac{\left(u^{2}-v^{2}\right)^{2}}{4 w^{2}}\left(\partial_{u u}+\partial_{v v}\right)-\partial_{v v}\right) \Psi=-\lambda_{2} \Psi, \quad L^{3} \Psi=\left(\partial_{u u}+\partial_{v v}\right) \Psi=0 .
\end{aligned}
$$

The separation equations are

$$
\left(\partial_{u u}+\lambda_{2}\right) \Psi^{(1)}=0, \quad\left(\partial_{v v}-\lambda_{2}\right) \Psi^{(2)}=0, \quad\left(\partial_{w w}-\frac{1}{w^{2}}-E\right) \Psi^{(3)}=0 .
$$

In terms of the $\Theta$ functions the final separated solution of $\Delta_{3} \Theta=E \Theta$ is

$$
\Theta=\frac{\Psi^{(1)}(u) \Psi^{(2)}(v) \Psi^{(3)}(w)}{w} .
$$


Example 5. Consider the Helmholtz equation in 3D Euclidean space $\Delta_{3} \Theta=E \Theta$. It is well known that this equation is regular separable in exactly eleven coordinate systems. Moreover true regular $R$-separation does not occur for any constant curvature space [15]. This example, due originally to Sym $[8,30,34,35]$ and presented from our point of view, shows that nonregular $R$-separation with two side conditions occurs in orthogonal coordinates distinct from the usual eleven. Consider Dupin-cyclidic coordinates $u, v, w$ such that

$$
\begin{aligned}
& x=\frac{b^{2} \cos u \cosh v+(c \cosh v-a \cos u) w}{a \cosh v-c \cos u}, \quad y=\frac{b \sin u(a \cosh v-w)}{a \cosh v-c \cos u}, \\
& z=\frac{b \sinh v(w-c \cos u)}{a \cosh v-c \cos u} .
\end{aligned}
$$

Here $a, b, c$ are positive parameters, with $b^{2}=a^{2}-c^{2}, c<a$, and $u \in[0,2 \pi)$. For any real $v$ we require $c \cos u<w<a \cosh v$, so that $x, y, z$ are real. Since

$$
\begin{aligned}
d s^{2} & =d x^{2}+d y^{2}+d z^{2}=\left(\frac{b(a \cosh v-w)}{a \cosh v-c \cos u}\right)^{2} d u^{2}+\left(\frac{b(w-c \cos u)}{a \cosh v-c \cos u}\right)^{2} d v^{2}+d w^{2} \\
& =H_{1}^{2} d u^{2}+H_{2}^{2} d v^{2}+H_{3}^{2} d w^{2},
\end{aligned}
$$

these coordinates are orthogonal. Thus

$$
\begin{aligned}
\Delta_{3} & =\partial_{x x}+\partial_{y y}+\partial_{z z} \\
& =\frac{1}{H_{1} H_{2} H_{3}}\left[\partial_{u}\left(H_{1}^{-1} H_{2} H_{3} \partial_{u}\right)+\partial_{v}\left(H_{1}^{1} H_{2}^{-1} H_{3} \partial_{v}\right)+\partial_{w}\left(H_{1} H_{2} H_{3}^{-1} \partial_{w}\right)\right] .
\end{aligned}
$$

We look for $R$-separable solutions

$$
\Theta=e^{R} \Psi=e^{R} \Psi^{(1)}(u) \Psi^{(2)}(v) \Psi^{(3)}(w), \quad e^{R}=\frac{1}{\sqrt{(w-c \cos u)(a \cosh v-w)}} .
$$

By direct calculation we can establish the operator identity

$$
e^{-R} \Delta_{3} e^{R}-E=H_{1}^{-2}\left(\partial_{u u}+\frac{1}{4}\right)+H_{2}^{-2}\left(\partial_{v v}-\frac{1}{4}\right)+H_{3}^{-2}\left(\partial_{w w}-E\right) .
$$

Thus the generalized Stäckel matrix and its inverse can be taken as

$$
S=\left(\begin{array}{ccc}
0 & 1 & 0 \\
0 & 0 & 1 \\
1 & -H_{1}^{-2} & -H_{2}^{-2}
\end{array}\right), \quad T=\left(\begin{array}{ccc}
H_{1}^{-2} & H_{2}^{-2} & 1 \\
1 & 0 & 0 \\
0 & 1 & 0
\end{array}\right),
$$

In terms of the $\Psi$ functions we have

$$
\begin{aligned}
& L^{1} \Psi=\left(H_{1}^{-2}\left(\partial_{u u}+\frac{1}{4}\right)+H_{2}^{-2}\left(\partial_{v v}-\frac{1}{4}\right)+\partial_{w w}\right) \Psi=E \Psi, \\
& L^{2} \Psi=\left(\partial_{u u}+\frac{1}{4}\right) \Psi=0, \quad L^{3} \Psi=\left(\partial_{v v}-\frac{1}{4}\right) \Psi=0 .
\end{aligned}
$$

The separation equations are

$$
\begin{aligned}
& \left(\partial_{u u}+\frac{1}{4}\right) \Psi^{(1)}=0, \quad\left(\partial_{v v}-\frac{1}{4}\right) \Psi^{(2)}=0, \quad\left(\partial_{w w}-E\right) \Psi^{(3)}=0, \\
& \Theta=\frac{\Psi^{(1)}(u) \Psi^{(2)}(v) \Psi^{(3)}(w)}{\sqrt{(w-c \cos u)(a \cosh v-w)}} .
\end{aligned}
$$

Here we have

$$
\left[L^{2}, L^{1}\right]=F_{22} L^{2}+F_{23} L^{3}, \quad\left[L^{3}, L^{1}\right]=F_{32} L^{2}+F_{33} L^{3}, \quad\left[L^{2}, L^{3}\right]=0,
$$

for first-order differential operators $F_{i j}$, so we have $R$-separation with two side conditions. 
Example 6. Again we consider the Helmholtz equation in 3D Euclidean space but now we choose coordinates $u, v, w$ such that

$$
x=\frac{1}{\sqrt{2}}(v+w) \cos u, \quad y=\frac{1}{\sqrt{2}}(v+w) \sin u, \quad z=\frac{1}{\sqrt{2}}(v-w) .
$$

We take a scalar potential

$$
\tilde{V}(x, y, z)=\frac{U(u)}{(v+w)^{2}}+V(v)+W(w)
$$

The metric is

$$
\begin{aligned}
& d s^{2}=d x^{2}+d y^{2}+d z^{2}=\frac{1}{2}\left[(v+w)^{2} d u^{2}+d v^{2}+d w^{2}\right] \\
& H_{u}^{-2}=\frac{2}{(v+w)^{2}}, \quad H_{v}^{-2}=H_{w}^{-2}=2 .
\end{aligned}
$$

Thus

$$
\begin{aligned}
\Delta_{3}+\tilde{V}= & \partial_{x x}+\partial_{y y}+\partial_{z z}+\tilde{V}=\frac{1}{H_{1} H_{2} H_{3}}\left[\partial_{u}\left(H_{1}^{-1} H_{2} H_{3} \partial_{u}\right)+\partial_{v}\left(H_{1}^{1} H_{2}^{-1} H_{3} \partial_{v}\right)\right. \\
& \left.+\partial_{w}\left(H_{1} H_{2} H_{3}^{-1} \partial_{w}\right)\right]+\frac{U(u)}{(v+w)^{2}}+V(v)+W(w) .
\end{aligned}
$$

We look for $R$-separable solutions

$$
\Theta=e^{R} \Psi=e^{R} \Psi^{(1)}(u) \Psi^{(2)}(v) \Psi^{(3)}(w), \quad e^{R}=\left(2\left[x^{2}+y^{2}\right]\right)^{-\frac{1}{4}} .
$$

By direct calculation we can establish the operator identity

$$
\begin{aligned}
e^{-R} \Delta_{3} e^{R}-E= & \frac{2}{(v+w)^{2}}\left(\partial_{u u}+U(u)+1\right)+2\left(\partial_{v v}+\frac{V(v)}{2}-\lambda_{2}\right) \\
& +2\left(\partial_{w w}+\frac{W(w)}{2}-\frac{E}{2}+\lambda_{2}\right) .
\end{aligned}
$$

Thus the generalized Stäckel matrix and its inverse can be taken as

$$
S=\left(\begin{array}{ccc}
\frac{1}{2} & 1 & 2-\frac{1}{(v+w)^{2}} \\
0 & -1 & -2 \\
0 & 0 & 1
\end{array}\right), \quad T=\left(\begin{array}{ccc}
2 & 2 & \frac{2}{(v+w)^{2}} \\
0 & -1 & -2 \\
0 & 0 & 1
\end{array}\right)
$$

The separation equations are

$$
\begin{aligned}
& \left(\partial_{u u}+U(u)+1\right) \Psi^{(1)}=0, \quad\left(\partial_{v v}+\frac{V(v)}{2}-\lambda_{2}\right) \Psi^{(2)}=0 \\
& \left(\partial_{w w}+\frac{W(w)}{2}-\frac{E}{2}+\lambda_{2}\right) \Psi^{(3)}=0, \quad \Theta=\frac{\Psi^{(1)}(u) \Psi^{(2)}(v) \Psi^{(3)}}{\sqrt{v+w}} .
\end{aligned}
$$

Example 7. As a generalization of the previous example, the Euclidean space metrics

$$
d s^{2}=\left(U_{1}(u) w+U_{2}(u) v+U_{3}(u)\right)^{2} d u^{2}+d v^{2}+d w^{2}
$$

for arbitrary functions $U_{j}(u)$ all lead to nonregular separation for Hamilton-Jacobi equations, and in the quantum case, to nonregular $R$-separation for velocity dependent potentials. The 
construction of separation equations and of the generalized Stäckel matrix is standard. As an instance, if

$$
x=F_{1}(u) v+F_{2}(u) w, \quad y=F_{3}(u) v+F_{4}(u) w, \quad z=F_{5}(u) v+F_{6}(u) w,
$$

for

$$
\begin{aligned}
& F_{1}=\sin ^{2} u, \quad F_{2}=\frac{-\cos ^{3} u}{\sqrt{1+\sin ^{2} u}}, \quad F_{3}=\sin u \cos u, \\
& F_{4}=\frac{\sin u\left(1+\cos ^{2} u\right)}{\sqrt{1+\sin ^{2} u}}, \quad F_{5}=\cos u, \quad F_{6}=\frac{-\sin ^{2} u}{\sqrt{1+\sin ^{2} u}},
\end{aligned}
$$

we have

$$
d s^{2}=d x^{2}+d y^{2}+d z^{2}=\left(\sqrt{1+\sin ^{2} u} v+\frac{\cos u\left(2+\sin ^{2} u\right)}{1+\sin ^{2} u} w\right)^{2} d u^{2}+d v^{2}+d w^{2} .
$$

Example 8. The negative constant curvature metric

$$
d s^{2}=\frac{d x^{2}+d y^{2}+d z^{2}}{z^{2}}=\frac{(u+v)^{2} d u^{2}+d v^{2}+d w^{2}}{w^{2}}
$$

for

$$
x=(u+v) \sin u+\cos u, \quad y=-(u+v) \cos u+\sin u, \quad z=w,
$$

leads to nonregular separation for Hamilton-Jacobi equations, and in the quantum case, to nonregular $R$-separation for velocity dependent potentials [7].

\section{Conclusions}

We have demonstrated that the characterization of symmetry related solutions modulo a side condition for Hamilton-Jacobi and Helmholtz or Schrödinger equations coincides with maximal nonregular separation of variables for which there is a generalized Stäckel matrix with one arbitrary column. We have also shown that these systems can be characterized geometrically, i.e., in a coordinate-free manner. We have demonstrated that there is a structure theory for this type of separation; it is not just a collection of examples. This allows us to obtain new separable solutions for these equations that cannot be obtained by the standard (regular) methods of separation of variables.

Our work leads to additional questions and possible extensions.

- Eisenhart showed that the Robertson condition for Helmholtz separabilty of a HamiltonJacobi regular separable coordinate system was the vanishing of the off-diagonal elements of the Ricci tensor in these coordinates $[12,14]$. Is there a corresponding geometrical interpretation of the obstruction problem for nonregular separation?

- Is there a physical interpretation of the side conditions?

- For regular separation of Hamilton-Jacobi and Helmholtz equations all separable systems on $N$-dimensional Euclidean spaces and $N$-spheres are known [15]. Can a similar classification of separation with a side condition be carried out?

- Much remains to be done in finding explicit, physically interesting, new solutions via this method.

- It is clear that separation with a side condition is also appropriate for heat, Laplace and wave equations, where the new solutions are likely to be more interesting physically. This should be done and a structure theory worked out. 


\section{Acknowledgements}

This work was partially supported by a grant from the Simons Foundation (\#208754 to Willard Miller, Jr.) and by the Australian Research Council (grant DP1095044 to G.E. Prince and P. Broadbridge).

\section{References}

[1] Arrigo D.J., Broadbridge P., Hill J.M., Nonclassical symmetry solutions and the methods of Bluman-Cole and Clarkson-Kruskal, J. Math. Phys. 34 (1993), 4692-4703.

[2] Benenti S., Chanu C., Rastelli G., Variable-separation theory for the null Hamilton-Jacobi equation, J. Math. Phys. 46 (2005), 042901, 29 pages.

[3] Benenti S., Francaviglia M., The theory of separability of the Hamilton-Jacobi equation and its applications to general relativity, in General Relativity and Gravitation, Vol. 1, Plenum, New York, 1980, 393-439.

[4] Bérubé J., Winternitz P., Integrable and superintegrable quantum systems in a magnetic field, J. Math. Phys. 45 (2004), 1959-1973, math-ph/0311051.

[5] Bluman G.W., Cole J.D., Similarity methods for differential equations, Applied Mathematical Sciences, Vol. 13, Springer-Verlag, New York, 1974.

[6] Bluman G.W., Cole J.D., The general similarity solution of the heat equation, J. Math. Mech. 18 (1969), 1025-1042.

[7] Cannon J.W., Floyd W.J., Kenyon R., Parry W.R., Hyperbolic geometry, in Flavors of Geometry, Math. Sci. Res. Inst. Publ., Vol. 31, Cambridge University Press, Cambridge, 1997, 59-115.

[8] Chanu C., Geometry of non-regular separation, in Symmetries and Overdetermined Systems of Partial Differential Equations, IMA Vol. Math. Appl., Vol. 144, Editors M. Eastwood, W. Miller Jr., Springer, New York, 2008, 305-317.

[9] Chanu C., Rastelli G., Fixed energy R-separation for Schrödinger equation, Int. J. Geom. Methods Mod. Phys. 3 (2006), 489-508, nlin.SI/0512033.

[10] Clarkson P.A., Mansfield E.L., Algorithms for the nonclassical method of symmetry reductions, SIAM J. Appl. Math. 54 (1994), 1693-1719.

[11] Degiovanni L., Rastelli G., Complex variables for separation of the Hamilton-Jacobi equation on real pseudoRiemannian manifolds, J. Math. Phys. 48 (2007), 073519, 23 pages, nlin.SI/0610012.

[12] Eisenhart L.P., Enumeration of potentials for which one-particle Schrödinger equations are separable, Phys. Rev. 74 (1948), 87-89.

[13] Eisenhart L.P., Riemannian geometry, 2nd ed., Princeton University Press, Princeton, N.J., 1949.

[14] Eisenhart L.P., Separable systems of Stäckel, Ann. of Math. (2) 35 (1934), 284-305.

[15] Kalnins E.G., Separation of variables for Riemannian spaces of constant curvature, Pitman Monographs and Surveys in Pure and Applied Mathematics, Vol. 28, Longman Scientific \& Technical, Harlow, 1986.

[16] Kalnins E.G., Miller Jr. W., Conformal Killing tensors and variable separation for Hamilton-Jacobi equations, SIAM J. Math. Anal. 14 (1983), 126-137.

[17] Kalnins E.G., Miller Jr. W., Intrinsic characterization of variable separation for the partial differential equations of mechanics, Atti Accad. Sci. Torino Cl. Sci. Fis. Mat. Natur. 117 (1983), suppl. 2, 511-533.

[18] Kalnins E.G., Miller Jr. W., Separation of variables on $n$-dimensional Riemannian manifolds. I. The $n$ sphere $S_{n}$ and Euclidean $n$-space $\mathbf{R}^{n}$, J. Math. Phys. 27 (1986), 1721-1736.

[19] Kalnins E.G., Miller Jr. W., The theory of orthogonal $R$-separation for Helmholtz equations, Adv. Math. 51 (1984), 91-106.

[20] Kalnins E.G., Miller Jr. W., Williams G.C., Recent advances in the use of separation of variables methods in general relativity, Philos. Trans. Roy. Soc. London Ser. A 340 (1992), 337-352.

[21] Levi D., Winternitz P., Nonclassical symmetry reduction: example of the Boussinesq equation, J. Phys. A: Math. Gen. 22 (1989), 2915-2924.

[22] Levi-Civita T., Sulla integrazione della equazione di Hamilton-Jacobi per separazione di variabili, Math. Ann. 59 (1904), 383-397. 
[23] Miller Jr. W., Mechanisms for variable separation in partial differential equations and their relationship to group theory, in Symmetries and Nonlinear Phenomena (Paipa, 1988), CIF Ser., Vol. 9, World Sci. Publ., Teaneck, NJ, 1988, 188-221.

[24] Miller Jr. W., The technique of variable separation for partial differential equations, in Nonlinear Phenomena (Oaxtepec, 1982), Lecture Notes in Phys., Vol. 189, Springer, Berlin, 1983, 184-208.

[25] Miller Jr. W., Kalnins E.G., Separation of variables methods for systems of differential equations in mathematical physics, in Lie Theory, Differential Equations and Representation Theory (Montreal, PQ, 1989), Univ. Montréal, Montreal, QC, 1990, 283-300.

[26] Nucci M.C., Clarkson P.A., The nonclassical method is more general than the direct method for symmetry reductions. An example of the FitzHugh-Nagumo equation, Phys. Lett. A 164 (1992), 49-56.

[27] Olver P.J., Rosenau P., Group-invariant solutions of differential equations, SIAM J. Appl. Math. 47 (1987), 263-278.

[28] Olver P.J., Rosenau P., The construction of special solutions to partial differential equations, Phys. Lett. A 114 (1986), 107-112.

[29] Ovsiannikov L.V., Group analysis of differential equations, Academic Press Inc., New York, 1982.

[30] Prus R., Sym A., Non-regular and non-Stäckel $R$-separation for 3-dimensional Helmholtz equation and cyclidic solitons of wave equation, Phys. Lett. A 336 (2005), 459-462.

[31] Shapovalov V.N., Separation of variables in a second-order linear differential equation, Differ. Equ. 16 (1981), 1212-1220.

[32] Shapovalov V.N., Stäckel spaces, Sib. Math. J. 20 (1979), 790-800.

[33] Stäckel P., Über die Integration der Hamilton-Jacobischen differential Gleichung mittelst Separation der Variabelen, Habilitationsschrift, Halle, 1891.

[34] Sym A., Solitons of wave equation, J. Nonlinear Math. Phys. 12 (2005), suppl. 1, 648-659.

[35] Sym A., Szereszewski A., On Darboux's approach to $R$-separability of variables, SIGMA 7 (2011), 095, 21 pages, arXiv:1102.2637.

[36] Thirring W., A course in mathematical physics. Vol. 3. Quantum mechanics of atoms and molecules, Lecture Notes in Physics, Vol. 141, Springer-Verlag, New York, 1981. 